\title{
Zur Dynamik prosozialen Verhaltens in einem asymmetrischen sozialen Dilemma: ein Beitrag zur experimentellen Spieltheorie The Dynamics of Pro-social Behavior in an Asymmetric Social Dilemma: A Behavioral Game-Theoretic Approach
}

\author{
Sonja Vogt ${ }^{*}$ \\ sonja.vogt@econ.uzh.ch \\ Werner Raub, Jeroen Weesie, Vincent Buskens \\ Institut für Soziologie/ICS, Universität Utrecht, Netherlands \\ w.raub@uu.nl; j.weesie@uu.nl; v.buskens@uu.nl
}

Institut für Volkswirtschaftslehre, Laboratory for Social and Neural Systems Research, Universität Zürich, Switzerland

\begin{abstract}
Zusammenfassung: In diesem Beitrag untersuchen wir prosoziales Verhalten im Sinne wechselseitiger Hilfeleistungen. Als formales Modell verwenden wir ein asymmetrisches wiederholtes Solidaritätsspiel zwischen zwei Akteuren. Wir modellieren Asymmetrie in drei Dimensionen: (1.) Nutzen, den ein Akteur aus der Hilfeleistung des anderen zieht, (2.) Kosten der eigenen Hilfeleistung und (3.) Wahrscheinlichkeit, mit der ein Akteur Hilfe benötigt. Wir untersuchen die Effekte von Asymmetrie auf die Dynamik wechselseitiger Hilfeleistungen im Verlauf des Spiels. Wir nehmen an, dass die Häufigkeit, mit der sich die Akteure helfen, vom Nutzen und den Kosten der Hilfeleistungen sowie von der Wahrscheinlichkeit abhängt, Hilfe zu benötigen. Unser Beitrag zur Theoriebildung ist ein einfaches adaptives Verhaltensmodell für die Erklärung wechselseitiger Hilfeleistungen, das an das Forschungsprogramm der experimentellen Spieltheorie („, behavioral game theory“) anschließt. Wir präsentieren zwei Varianten eines solchen Modells: eine sozialpsychologisch inspirierte Variante und eine Variante, die auf spieltheoretischen Verhandlungsmodellen beruht. Unser Modell ist robust in dem Sinn, dass beide Varianten zu ähnlichen Vorhersagen führen. Empirische Daten aus zwei Laborexperimenten bestätigen diese Vorhersagen.
\end{abstract}

Schlagworte: Adaptives Verhalten; Equity-Theorie; Experimentelle Spieltheorie; Kalai-Smorodinsky-Lösung; Prosoziales Verhalten; Reziprozität; Solidaritätsspiel; Verhandlungsmodelle; Wiederholte Spiele.

Summary: This contribution examines pro-social behavior in social support relations. Such relations are modeled as an asymmetric and repeated social dilemma game in which two actors can provide support for each other. We allow for asymmetry in three dimensions: (1) benefits from receiving support, (2) costs of providing support, and (3) the likelihood of needing support. Theoretically and empirically, we analyze the effects of asymmetry on the dynamics of support. We assume that the costs of providing and the benefits of receiving support as well as the likelihood of needing support affect an actor's willingness to provide support. Using a behavioral game theory approach, we contribute to theory formation by developing a simple adaptive model for explaining behavior in support relations. More precisely, we offer two variants of such an adaptive model. One variant is inspired by social psychology and implements assumptions from equity theory. The other variant is inspired by bargaining theory and uses the Kalai-Smorodinsky bargaining solution. Our model is robust in the sense that both variants lead to similar predictions. Data from laboratory experiments confirm these predictions.

Keywords: Adaptive Behavior; Bargaining Theory; Behavioral Game Theory; Equity Theory; Kalai-Smorodinsky Bargaining Solution; Pro-social Behavior; Reciprocity; Repeated Games; Support Game.

* Zwei Gutachtern und den Herausgebern der ZfS dan-
ken wir für nützliche Hinweise. Für finanzielle Unterstüt-
zung durch das RUBICON-Projekt „Fairness in Hetero-
geneous Social Dilemmas“ (446-07-024; Vogt) und das
PIONIER-Programm „The Management of Matches“
(PGS 50-370 und S96-168; Raub und Weesie) danken
wir der Niederländischen Organisation für wissenschaft-
liche Forschung (NWO). Ebenso danken wir der Univer-
sität Utrecht für finanzielle Unterstützung durch das High
Potential-Programm „Dynamics of Cooperation, Net- works, and Institutions“ (Buskens). Für finanzielle Unterstützung durch das Projekt „The Social Dynamics of Normative Behavior“ (100014-130127; Efferson und Vogt) danken wir dem Schweizerischen Nationalfonds zur Förderung der wissenschaftlichen Forschung (SNF). Unser Beitrag setzt eine frühere gemeinsame Arbeit (Weesie et al. 2007) fort. Im hier vorliegenden Beitrag verwenden wir einen modifizierten theoretischen Ansatz, zusätzliche empirische Daten (Experiment 2) und eine andere Analysestrategie. 


\section{Einleitung}

Frau Neumann und Frau Morgenstern sind Arbeitskolleginnen. Beide haben eine Teilzeitstelle als Krankenschwester. Sie können gegenseitig ihre Kinder betreuen, während jeweils die andere arbeitet. Nehmen wir zunächst eine symmetrische Situation an: Beide haben ein zweijähriges Kind, erhalten den gleichen Lohn und arbeiten an gleich vielen, wenn auch unterschiedlichen Tagen. Unter welchen Bedingungen werden sie einander helfen und wie oft werden sie dies tun? Man könnte sich z. B. vorstellen, dass sie einander gleich häufig helfen, z. B. immer, wenn eine von ihnen arbeitet und daher Hilfe bei der Kinderbetreuung benötigt. Nehmen wir demgegenüber an, dass sich die beiden Frauen in verschiedenen Hinsichten unterscheiden: Frau Neumann hat Zwillinge während Frau Morgenstern ein Einzelkind hat; Zwillinge benötigen mehr Aufmerksamkeit als ein einzelnes Kind. Frau Morgenstern kann also nur wenig Hausarbeit verrichten, während sie die Kinder von Frau Neumann betreut. Die Kosten der Hilfeleistung sind dadurch für Frau Morgenstern höher als für Frau Neumann. Darüber hinaus arbeitet Frau Neumann fünf Stunden mehr pro Woche als Frau Morgenstern. Frau Neumann benötigt daher auch häufiger Hilfe als Frau Morgenstern. Außerdem arbeitet Frau Neumann auf der Intensivstation und bekommt ein höheres Gehalt als Frau Morgenstern. Frau Neumann zieht also zusätzlich einen höheren Nutzen aus der gegenseitigen Kinderbetreuung. Bei solchen Unterschieden stellt sich nicht nur die Frage, unter welchen Bedingungen und wie häufig die Frauen einander helfen. Es drängt sich vielmehr auch die Frage auf, ob die beiden Frauen einander mit gleicher Häufigkeit helfen oder ob eine der beiden häufiger Hilfe leistet als die andere. Es wäre z. B. verständlich, dass Frau Neumann ziemlich häufig hilft, wenn Frau Morgenstern Hilfe benötigt, denn die Kosten der Hilfeleistung sind für Frau Neumann geringer und zudem benötigt Frau Morgenstern seltener Hilfe. Frau Morgenstern hilft hingegen möglicherweise weniger häufig, da Frau Neumann öfter Hilfe benötigt und die Hilfeleistung für Frau Morgenstern kostspieliger ist.

In diesem Beitrag untersuchen wir prosoziales Verhalten im Sinne wechselseitiger Hilfe in dauerhaften Beziehungen zwischen im angedeuteten Sinn asymmetrischen Akteuren. Wir wollen die Frage beantworten, wie sich die Dynamik wechselseitiger Hilfe über die Zeit hinweg entwickelt. Welche Rolle spielen dabei Größen wie die Kosten und der Nutzen der Hilfeleistung oder die Häufigkeit der Hilfs- bedürftigkeit? Wie wird prosoziales Verhalten durch entsprechende Asymmetrien zwischen den Akteuren beeinflusst? In symmetrischen Interaktionen liegt die Annahme nahe, dass Akteure sich gleich häufig helfen (Fehr \& Gintis 2007). Für asymmetrische Interaktionen ist diese Annahme aber viel weniger naheliegend und theoretische Modelle für die Analyse solcher Fälle fehlen weitestgehend. Wir tragen zur Schließung dieser Forschungslücke bei und entwickeln ein einfaches adaptives Verhaltensmodell für die Erklärung wechselseitiger Hilfeleistungen, das an das Forschungsprogramm der experimentellen Spieltheorie (,,behavioral game theory"; Camerer 2003) anschließt.

Wir werden zeigen, dass wechselseitige Hilfeleistung ein Kooperationsproblem in dem Sinn ist, dass sie für die beteiligten Akteure vorteilhafter ist als eine Situation, in der keine Hilfe geleistet wird, dass jeder der beteiligten Akteure aber auch einen individuellen Anreiz hat, einseitig den Kosten der eigenen Hilfeleistung aus dem Weg zu gehen. ${ }^{1}$ Unterschiede zwischen Akteuren hinsichtlich des Nutzens aus erhaltener Hilfe, der Kosten der Hilfeleistung und der Wahrscheinlichkeit der Hilfsbedürftigkeit betreffen dagegen zentrale Dimensionen sozialer Ungleichheit. Unser Beispiel betrifft Effekte der Ungleichheit zwischen Arbeitskollegen: Wie wirken sich Unterschiede zwischen Kollegen im Hinblick auf ihre Kenntnisse und Fähigkeiten, die Häufigkeit, mit der sie Hilfe benötigen, ihre Zeitrestriktionen, ihre Entlohnung usw. auf die Bereitschaft zur wechselseitigen Hilfe aus? Wir schließen damit an die klassische Studie von Blau (1963) über wechselseitige Hilfeleistungen bei Arbeitsproblemen zwischen Organisationsmitgliedern an, die sich hinsichtlich ihrer Kompetenzen unterscheiden. ${ }^{2}$ Ein anderer Anwendungsfall sind wechselseitige Hilfeleistungen in informellen sozialen Beziehungen zwischen Freunden und Bekannten oder zwischen Nachbarn. Wie wirken sich in solchen Beziehungen Unterschiede der angedeuteten Art auf prosoziales Verhalten und "Solidarität“ zwischen den Akteuren aus? In diesem Sinn widmet sich unser Beitrag einer Schnittstelle von zwei zentralen Forschungsbereichen der

\footnotetext{
${ }^{1}$ Vgl. Voss 1985 und Raub \& Voss 1986 für eine systematische Rekonstruktion des Zusammenhangs zwischen solchen Kooperationsproblemen und dem klassischen soziologischen Problem der sozialen Ordnung.

${ }^{2}$ Blau betrachtet den Tausch von Hilfeleistungen bei der Lösung von Arbeitsproblemen gegen soziale Anerkennung. In unserer Analyse geht es dagegen - in der TauschTerminologie - um den Tausch der heutigen eigenen Hilfeleistung gegen vorangegangene oder zukünftige Hilfe seitens des Partners.
} 
Soziologie, nämlich einerseits Effekten sozialer Ungleichheit auf individuelles Verhalten und andererseits den Bedingungen von Kooperation bei Anreizproblemen.

Unser Beitrag ist im Forschungsprogramm der experimentellen Spieltheorie angesiedelt (vgl. Camerer 2003). Dieses Forschungsprogramm verknüpft formale spieltheoretische Modellierungen ${ }^{3}$ mit Annahmen über Verhaltensregelmäßigkeiten aus der (Sozial-) Psychologie. Die experimentelle Spieltheorie zielt außerdem auf die systematische Verknüpfung von theoretischer Modellierung und empirischer experimenteller Forschung. Diekmann (2009: Kap. 10) bietet eine knappe und instruktive Übersicht zur experimentellen Spieltheorie, in der er hervorhebt, dass sich dieses Forschungsprogramm in neuerer Zeit zwar vor allem in der experimentellen Wirtschaftsforschung entwickelt hat und in der Ökonomik inzwischen sehr einflussreich geworden ist, experimentelle Untersuchungen gleichwohl in der Soziologie mehr noch als in der Ökonomik eine lange und reiche Tradition haben. Diekmann zeigt mit vielen Beispielen, dass die experimentelle Spieltheorie sowohl theoretisch als auch empirisch neue Einsichten zu klassischen soziologischen Fragestellungen bietet und dass es in diesem Forschungsprogramm in vielen Hinsichten um eine systematische Integration von Soziologie, Ökonomik und empirischer experimenteller Forschung geht. Der vorliegende Beitrag schließt genau dort an.

Nicht zuletzt durch die Entwicklung der experimentellen Spieltheorie werden experimentelle Methoden inzwischen nicht nur in der (Sozial-) Psychologie, sondern vermehrt auch in anderen Sozialwissenschaften angewendet. Laborexperimente dienen vor allem der systematischen Prüfung von Hypothesen auf der Grundlage einer Kontrolle unabhängiger Variablen und der Repräsentation theoretischer Annahmen in experimentellen Bedingungen. Laborexperimente sind oft „künstlich“, und das führt zu der Frage, ob experimentelle Resultate generalisierbar sind. Falk \& Heckman (2009) zeigen im Detail, dass die „Künstlichkeit“ von Laborexperimenten nicht notwendigerweise impliziert, dass die zu prüfenden kausalen Effekte mit anderen Forschungsdesigns besser isoliert werden können. Unabhängig davon spricht natürlich vieles dafür, Laborexperimente mit anderen Forschungsdesigns wie z. B. Feldexperimenten aber auch Surveys und

\footnotetext{
${ }^{3}$ Vgl. für Übersichten und als Einführung mit soziologischen Anwendungsbeispielen und weiterführenden Literaturhinweisen Raub \& Buskens 2006 sowie Diekmann 2009.
}

Vignettenstudien zu kombinieren und durch Kreuzvalidierungen den bekannten Problemen und Fehlerquellen der unterschiedlichen Designs zu begegnen. ${ }^{4}$

Der vorliegende Beitrag ist folgendermaßen aufgebaut: Zunächst stellen wir eine spieltheoretische Modellierung prosozialen Verhaltens im Sinne wechselseitiger Hilfeleistungen in einem asymmetrischen und wiederholten sozialen Dilemma vor. Danach skizzieren wir den spieltheoretischen Standardansatz für die Ableitung von Hypothesen über prosoziales Verhalten in diesem Spiel und zeigen, in welchen Hinsichten dieser Ansatz unbefriedigend ist. Als Alternative entwickeln wir ein einfaches adaptives Verhaltensmodell für die Erklärung wechselseitiger Hilfeleistungen. Wir präsentieren zwei Varianten dieses Modells: eine sozialpsychologische Variante, die auf Annahmen aus der Equity-Theorie aufbaut, und eine Variante, die durch spieltheoretische Verhandlungsmodelle motiviert ist und auf der Kalai-Smorodinsky-Lösung beruht. Mittels experimenteller Daten überprüfen wir die Vorhersagen, die aus diesen beiden Varianten folgen. Eine zusammenfassende Diskussion und Vorschläge für weitere Forschung schließen den Beitrag ab.

\section{Theoretische Modellierung von prosozialem Verhalten}

Wir verwenden ein spieltheoretisches Modell für Hilfebeziehungen: das Solidaritätsspiel (vgl. Weesie 1988: 155-160; Hegselmann 1994; Vogt \& Weesie 2004). ${ }^{5}$ Dieses Spiel (siehe Abbildung 1) wird von zwei Akteuren A und B gespielt. Ein Zufallszug (in spieltheoretischer Terminologie: ein Zug der „Natur") entscheidet darüber, ob Akteur A oder Akteur B Hilfe benötigt. Wir nehmen an, dass Akteur $i(i=$ A, B) mit Wahrscheinlichkeit $\pi_{i}$ Hilfe benötigt, dass jeder Akteur mit positiver Wahrscheinlichkeit hilfsbedürftig wird $\left(0<\pi_{i}<1\right.$ für $\left.i=\mathrm{A}, \mathrm{B}\right)$ und dass jeweils genau ein Akteur Hilfe benötigt $\left(\pi_{A}+\pi_{B}=1\right)$. In einem komplexeren Modell könnte man leicht berücksichtigen, dass möglicherweise beide Akteure

\footnotetext{
${ }^{4}$ Vgl. Falk \& Heckman 2009 sowie Gächter \& Thöni 2011 für neue und informative Diskussionen der Vor- und Nachteile experimenteller Methoden; vgl. Raub \& Buskens 2008 für ein Beispiel der Verwendung komplementärer empirischer Designs zur wiederholten Prüfung derselben Hypothesen in einem Forschungsfeld, das mit den Fragestellungen des vorliegenden Beitrags verwandt ist.

${ }^{5}$ Vgl. Diekmann (2009) für eine zugängliche und zuverlässige Einführung spieltheoretischer Begriffe und Annahmen, die wir in diesem Beitrag verwenden.
} 
hilfsbedürftig sind oder aber keiner der beiden Akteure Hilfe benötigt. Unsere Resultate sind robust relativ zu solchen Komplikationen und wir können uns daher mit dem einfacheren Modell begnügen. Der selbst nicht hilfsbedürftige Akteur muss entscheiden, ob er dem hilfsbedürftigen Akteur hilft oder nicht. Die Hilfeleistung ist mit Kosten $c_{i}>0$ für den Hilfe leistenden Akteur verbunden. Wir nehmen an, dass diese Kosten geringer sind als der Nutzen $b_{i}$, den der Akteur aus erhaltener Hilfe zieht. ${ }^{6}$ Ohne Verlust an Allgemeinheit nehmen wir an, dass jeder Akteur die Auszahlung 0 erhält, wenn keine Hilfe geleistet wird. Das Spiel ist symmetrisch, wenn sich die Akteure hinsichtlich Kosten, Nutzen und Wahrscheinlichkeit der Hilfsbedürftigkeit nicht unterscheiden, wenn also $\pi_{A}=\pi_{B}$, $c_{A}=c_{B}$ und $b_{A}=b_{B}$, andernfalls ist das Spiel asymmetrisch. In einem symmetrischen Spiel sprechen wir auch von symmetrischen Akteuren, in einem asymmetrischen Spiel von asymmetrischen Akteuren.

Wir nehmen an, dass das Solidaritätsspiel ein nichtkooperatives Spiel ist. Das bedeutet, dass bindende Vereinbarungen zwischen den Akteuren und bindende einseitige Verpflichtungen eines Akteurs ausgeschlossen sind. ${ }^{7}$ Nicht zu helfen, ist dann wegen $c_{i}>0$ für beide Akteure die dominante Strategie (nicht zu helfen, ist unabhängig von der Strategie des anderen Akteurs mit einer höheren Auszahlung verbunden als zu helfen). Mithin ist wechselseitige Verweigerung von Hilfe das einzige Nash-Gleichgewicht (jeder Akteur maximiert seine eigene Auszahlung, gegeben die Gleichgewichtsstrategie des anderen Akteurs). Wechselseitige Hilfeleistung stellt bei-

\footnotetext{
${ }^{6}$ Man beachte, dass wir Kosten und Nutzen für denselben Akteur $i$ miteinander vergleichen und dass wir keine Annahmen über interpersonelle Nutzenvergleiche benötigen. Genau genommen sind die Parameter $c_{i}$ und $b_{i}$ Nutzendifferenzen. Der Parameter $c_{i}$ modelliert die Differenz zwischen dem Nutzen von $i$, wenn er selbst keine Hilfe benötigt und keine Hilfe leistet, und dem Nutzen von $i$, wenn er selbst keine Hilfe benötigt, aber Hilfe leistet. Entsprechend ist $b_{i}$ die Differenz zwischen dem Nutzen von $i$, wenn er selbst Hilfe benötigt und erhält, und dem Nutzen von $i$, wenn er selbst Hilfe benötigt, aber keine Hilfe erhält.

${ }^{7}$ Mit der Annahme, dass das Solidaritätsspiel ein nichtkooperatives Spiel im Standardsinn der Spieltheorie ist, wird nicht ausgeschlossen, dass sich die Akteure kooperativ in dem Sinn verhalten, dass sie Hilfe leisten. Modelle für nichtkooperative Spiele werden gerade dazu verwendet, um Bedingungen für kooperatives Verhalten zu analysieren, und genau das tun wir auch im vorliegenden Beitrag.
}

de Akteure besser, wenn der erwartete Nutzen erhaltener Hilfe größer ist als die erwarteten Kosten der eigenen Hilfeleistung, wenn also $\pi_{i} b_{i}-\pi_{j} c_{i}>0$ für $i=\mathrm{A}, \mathrm{B}$ und $j \neq i$. Das Solidaritätsspiel ist dann ein soziales Dilemma in dem Sinn, dass individuell rationales Verhalten, nämlich spieltheoretisches Gleichgewichtsverhalten (nicht zu helfen), und kollektive Rationalität (wechselseitige Hilfe, die beide Akteure besser stellt) konfligieren (vgl. Rapoport 1974; Raub \& Voss 1986). Wir nehmen im Folgenden an, dass die Dilemma-Bedingung erfüllt ist.

Prosoziales Verhalten ${ }^{8}$ treffen wir typischerweise in dauerhaften sozialen Beziehungen an. Wechselseitige Hilfeleistungen in solchen Beziehungen können wir daher auch als ein paradigmatisches Beispiel für sozialen Tausch betrachten (Blau 1964, 1968; Emerson 1976; Kollock 1998). Frau Neumann und Frau Morgenstern benötigen mehr oder weniger regelmäßig die Hilfe der Kollegin und stehen selbst mehr oder weniger regelmäßig vor der Entscheidung, Hilfe zu leisten oder zu unterlassen. Wir modellieren diesen aus soziologischer Sicht wesentlichen Aspekt der Dauerhaftigkeit wechselseitiger Hilfebeziehungen im iterierten Solidaritätsspiel (IS). Nachdem das Solidaritätsspiel zum Zeitpunkt $t(t=1,2, \ldots)$ gespielt wurde, wird das gleiche Spiel mit einer Wahrscheinlichkeit $w(0<w<1)$ zum Zeitpunkt $t+1$ erneut gespielt. Mit der komplementären Wahrscheinlichkeit $1-w$ endet das Spiel nach „Runde“ $t$. Das IS ist somit die unbestimmt häufig wiederholte Version des Solidaritätsspiels. Die Parameter des Solidaritätsspiels bleiben konstant über alle Runden $t$. Die Parameter des Spiels in Runde $t$ hängen also nicht davon ab, was in früheren Runden $1, \ldots, t-1$ geschehen ist. Beispielsweise hängt die Wahrscheinlichkeit, Hilfe zu benötigen, nicht davon ab, wie häufig ein Akteur bereits in der Vergangenheit geholfen oder selbst Hilfe erhalten hat. ${ }^{9}$

\footnotetext{
${ }^{8}$ Vgl. z. B. Bierhoff 2002 für eine Übersicht, die deutlich macht, dass das Solidaritätsspiel zentrale Merkmale prosozialen Verhaltens repräsentiert.

9 „Häufiger helfen“ interpretieren wir im IS in dem Sinn, dass Frau Neumann mit einer größeren Wahrscheinlichkeit Frau Morgenstern hilft als umgekehrt. Wir untersuchen also probabilistisches Verhalten. „Häufiger helfen“ könnten wir demgegenüber auch interpretieren als „Frau Neumann hilft den ganzen Tag, während Frau Morgenstern nur einige Stunden hilft" (vgl. Weesie 2006 für eine Analyse dieser Variante).
} 


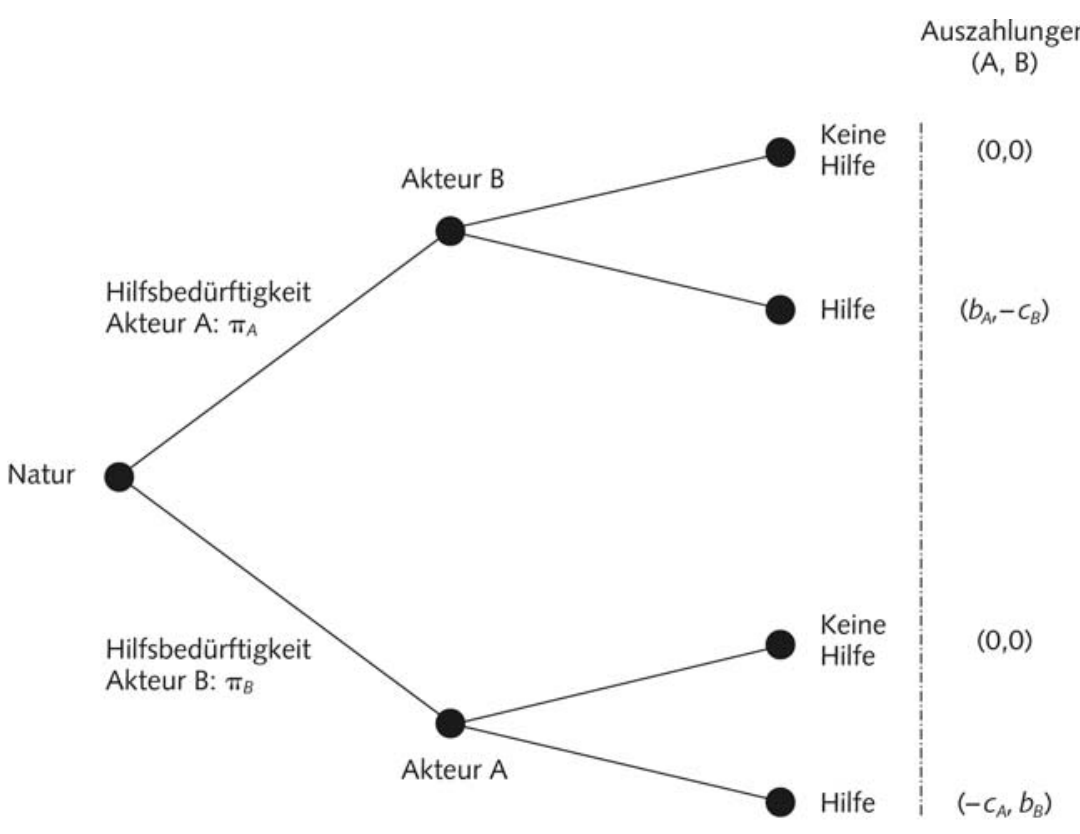

Abb. 1 Extensive Form des Solidaritätsspiels mit $b_{i}>c_{i}>0, i=\mathrm{A}, \mathrm{B}$.

\subsection{Der spieltheoretische Standardansatz für die Ableitung von Hypothesen über prosoziales Verhalten im iterierten Solidaritätsspiel}

Die moderne Spieltheorie bietet einen „Standardansatz" für die Analyse des IS und für die Ableitung von Hypothesen über wechselseitige Hilfeleistungen. Im Kern geht es bei diesem Ansatz um Hilfeleistungen als Resultat bedingt kooperativer und in diesem Sinn reziproker Strategien (Gouldner 1960; Axelrod 1984; Taylor 1987; Raub \& Voss 1986; Diekmann 2004). Erhaltene Hilfe wird dadurch belohnt, dass man selbst Hilfe leistet, wenn der Partner in der Zukunft Hilfe benötigt, während verweigerte Hilfe dadurch bestraft wird, dass man selbst in der Zukunft Hilfe verweigert, wenn der Partner sie benötigt. Es lässt sich zeigen, dass das IS Gleichgewichte in der Art haben kann, dass beide Akteure bedingt kooperative Strategien verwenden (Vogt \& Weesie 2004). Wenn beide Akteure solche Strategien verwenden, dann wird in jeder Runde Hilfe geleistet. Wechselseitige Hilfeleistung wird also in diesem Ansatz als individuell rationales Gleichgewichtsverhalten konzeptualisiert und ist Resultat „aufgeklärten Selbstinteresses“: Wenn Akteur $i$ antizipiert, dass $j$ eine bedingt kooperative Strategie verwendet, dann muss $i$ den kurzfristigen Anreiz zur Vermeidung der eigenen Kooperationskosten gegen die langfristigen Nachteile durch verweigerte zukünftige Hilfeleistungen des Partners abwägen.
Ein Modell bedingter Kooperation im IS, das diesem Standardansatz folgt, betrachtet Gleichgewichte von so genannten Trigger-Strategien (vgl. Diekmann 2009: Kap. 7.4). Im IS verlangt die TriggerStrategie, dass ein Akteur dem Partner immer hilft, solange der Partner niemals Hilfe verweigert hat. Wenn aber der Partner einmal Hilfe verweigert hat, hilft der Akteur ihm niemals wieder. Die TriggerStrategie ist ersichtlich eine bedingt kooperative Strategie im angedeuteten Sinn und wenn beide Akteure diese Strategie verwenden, dann werden sie einander im IS ständig wechselseitig helfen. Man kann zeigen (vgl. Vogt \& Weesie 2004), dass Trigger-Strategien genau dann ein Gleichgewicht im IS sind, wenn die Fortsetzungswahrscheinlichkeit $w$ groß genug ist, nämlich für jeden der beiden Akteure $i$ einen Schwellenwert nicht unterschreitet, der von den Kosten geleisteter $\left(c_{i}\right)$ und dem Nutzen erhaltener Hilfe $\left(b_{i}\right)$ abhängt sowie von der Wahrscheinlichkeit der eigenen Hilfsbedürftigkeit $\left(\pi_{i}\right)$. Empirisch prüfbare Hypothesen folgen aus der komparativ-statischen Analyse der Gleichgewichtsbedingung. Man untersucht dann, bei welchen Änderungen der Parameter des IS die Gleichgewichtsbedingung restriktiver oder aber weniger restriktiv wird, und leitet Hypothesen unter der zusätzlichen Annahme ab, dass Hilfeleistungen wahrscheinlicher werden, wenn die Gleichgewichtsbedingungen für Trigger-Strategien weniger restriktiv ausfallen (vgl. Raub \& Buskens 2006: 569-570). So gelangt 
man z. B. zu Hypothesen, dass Hilfeleistungen wahrscheinlicher werden, wenn die Fortsetzungswahrscheinlichkeit zunimmt, wenn die Kosten der Hilfeleistung abnehmen und wenn der Nutzen erhaltener Hilfe zunimmt. Komplexere und intuitiv weniger nahe liegende Hypothesen betreffen z. B. Effekte zunehmender Asymmetrie zwischen den Akteuren. Diese Hypothesen wurden auch bereits experimentell überprüft (Vogt \& Weesie 2006).

Dieser Ansatz ist analytisch elegant und führt zu prüfbaren Hypothesen, aber er ist in verschiedenen Hinsichten auch unbefriedigend. Insbesondere kann er nicht erklären, dass möglicherweise einer der beiden Akteure häufiger als der andere Hilfe leistet. In einem asymmetrischen Spiel ist es aber keineswegs intuitiv nahe liegend, dass beide Akteure die gleiche Strategie verwenden und einander gleich häufig, nämlich immer, helfen. Die angedeutete Gleichgewichtsbedingung für Trigger-Strategien berücksichtigt sehr wohl Asymmetrie zwischen den Akteuren, aber sie tut das in der Weise, dass jeder der beiden Schwellenwerte von den individuellen Parametern von einem der beiden Akteure abhängt. Asymmetrie impliziert dann unterschiedliche Schwellenwerte. Da die Existenz eines Gleichgewichts in Trigger-Strategien vom Maximum der beiden Schwellenwerte abhängt, beeinflusst Asymmetrie die Gleichgewichtsbedingung. Im Gleichgewicht sollten aber beide Akteure die gleiche Strategie verwenden. Wenn jedoch z. B. Akteur A doppelt so häufig wie Akteur B Hilfe benötigt, ist es wenig plausibel, dass beide Akteure einander ständig helfen. Man könnte sich eher vorstellen, dass Akteur A häufiger Hilfe leistet, wenn B Hilfe benötigt, als B, wenn A Hilfe benötigt. Experimentelle Evidenz bestätigt derartige Vermutungen (Vogt et al. 2006).

Man kann den spieltheoretischen Standardansatz verfeinern und komplexere Trigger-Strategien berücksichtigen. Man betrachte z. B. eine Strategie, derzufolge Akteur $i$ mit Wahrscheinlichkeit $\alpha_{i}$ hilft, falls $j$ Hilfe benötigt und falls $j$ in der Vergangenheit mindestens mit Wahrscheinlichkeit $\alpha_{j}$ geholfen hat, wenn $i$ Hilfe benötigte. Abweichungen von $j$ von dieser Regel würde $i$ wiederum mit Verweigerung von Hilfe in allen zukünftigen Runden sanktionieren. Man kann Gleichgewichtsbedingungen für solche komplexeren Trigger-Strategien herleiten und erhält dann im Prinzip auch ein spieltheoretisches Modell, das für manche, aber nicht für alle Runden im IS Hilfeleistungen vorhersagt und aus dem auch folgt, dass sich die Akteure hinsichtlich der Wahrscheinlichkeiten unterscheiden, mit denen sie Hilfe leisten, da $0<\alpha_{i}<1$ und $\alpha_{i} \neq \alpha_{j}$ für $i=\mathrm{A}$, B gelten kann (vgl. Weesie 2006). Ein solches Modell enthält jedoch die problematische Annahme, dass die Akteure nicht nur das Verhalten des Partners beobachten können, sondern auch die Wahrscheinlichkeit, mit der ein Partner hilft. Es gibt Modelle, die die Annahme umgehen, dass solche Wahrscheinlichkeiten beobachtbar sind, aber diese erfordern entweder die Verwendung sehr starker Rationalitätsannahmen (Fudenberg \& Maskin 1986) oder die Untersuchung des Falles, dass Akteure nicht mit einer bestimmten Wahrscheinlichkeit, sondern in einem bestimmten Umfang (z. B. für eine bestimmte Dauer) Hilfe leisten (vgl. Fußnote 9). Außerdem gibt es viele verschiedene Gleichgewichte, in denen einer der Akteure mit Wahrscheinlichkeit $\alpha_{i}$ und der andere mit Wahrscheinlichkeit $\alpha_{j}$ Hilfe leistet. Man würde also zusätzliche Argumente für die Auszeichnung eines dieser Gleichgewichte als „Lösung “ des Spiels benötigen.

\subsection{Experimentelle Spieltheorie}

Camerer (2003: 465) charakterisiert die experimentelle Spieltheorie („,behavioral game theory“) als „a formal modification of rational game theory aided by experimental evidence and psychological intuition. The modifier , behavioral' is a reminder that the theory is explicitly intended to predict behavior of people (and collectivities like firms), and draws as directly as possible on evidence from psychology to do so“. Man kann zwei Formen der „Modifikation “ der Spieltheorie unterscheiden: Manche Ansätze der experimentellen Spieltheorie halten an der Rationalitätsannahme selbst fest, nehmen also Gleichgewichtsverhalten an, modifizieren aber andere „Standardannahmen“ der Spieltheorie wie etwa die Annahme hinsichtlich der Nutzenfunktion, dass Akteure ausschließlich an der Maximierung der eigenen materiellen Auszahlungen interessiert seien (z. B. „eigener Nutzen = eigene monetäre Auszahlung“). Einflussreiche Modelle der Ungleichheitsaversion wie das von Fehr \& Schmidt (1999), das ERC-Modell von Bolton \& Ockenfels (2000; Ockenfels 1999) und die umfangreiche experimentelle Forschung zu diesen Modellen sind Repräsentanten eines solchen Ansatzes. In diesen Modellen wird angenommen, dass Akteure nicht nur durch ihre eigene monetäre Auszahlung motiviert sind, sondern zugleich auch Fairnesskriterien berücksichtigen. Man schließt also bei (sozial-) psychologischer und soziologischer Forschung über soziale Vergleichsprozesse an und arbeitet mit einer komplexeren Nutzenfunktion, während man die Annahme des Gleichgewichtsverhaltens beibehält. Andere Ansätze der experimentellen Spieltheorie 
geben (auch) die Annahme des Gleichgewichtsverhaltens auf und ersetzen sie durch Annahmen, die sich - in einem weiten Sinn - auf begrenzt rationales Verhalten beziehen und von denen man sich erhofft, dass sie sich empirisch besser bewähren als die strengeren Rationalitätsannahmen der Standardspieltheorie (vgl. Camerer 2003).

Wir folgen dieser zweiten Art von Ansätzen und verwenden im Folgenden ein adaptives Verhaltensmodell, mit dem man vorhersagen kann, wer häufiger Hilfe leistet und wie sich wechselseitige Hilfeleistungen über die Zeit hinweg entwickeln. Das Modell unterstellt einen einfachen „Matching“Mechanismus (Herrnstein 1977). Der Grundgedanke ist, dass das Verhalten der Akteure von zwei Größen abhängt. Eine dieser beiden Größen ist die Zielgröße. Diese spezifiziert, wie häufig ein Akteur bereit sein sollte, zu helfen, falls der Partner Hilfe benötigt. Wir nehmen an, dass die Akteure in jeder Runde des IS ihre Zielgröße mit einer zweiten Größe vergleichen, nämlich der Erfahrungsgröße, die in einfacher Weise aus der "Geschichte“ des IS folgt, also aus dem Verhalten der beiden Akteure in den vergangenen Runden. Unser Modell ist also „backward-looking“. Spieltheoretisches Gleichgewichtsverhalten ist "forward-looking“ in dem Sinn, dass Verhalten getrieben wird durch rationale Antizipation der Folgen des Verhaltens. Backward-looking Modelle wie das hier verwendete nehmen dagegen an, dass Verhalten durch Erfahrungen aus der Vergangenheit und durch Regeln geleitet wird, die heutiges Verhalten von früheren Erfahrungen abhängig machen. In der Forschung über soziale Dilemmata werden solche Modelle seit einiger Zeit als Alternativen zum spieltheoretischen Ansatz verwendet (vgl. z. B. Macy \& Flache 2002). Unser MatchingMechanismus ist eine neue Variante solcher backward-looking Modelle.

\subsubsection{Annahmen zur Verhaltensdynamik}

Die Zielgröße ist ein Quotient, der angibt, wie oft jeder Akteur Hilfe leistet, falls der Partner Hilfe benötigt. Wir definieren die Zielgröße der Akteure A und $\mathrm{B}$ als das Verhältnis der bedingten Wahrscheinlichkeit $\alpha_{A}$, dass A hilft, gegeben B benötigt Hilfe, zur bedingten Wahrscheinlichkeit $\alpha_{B}$, dass B hilft, gegeben A benötigt Hilfe:

$$
s_{A B}=\frac{\alpha_{A}}{\alpha_{B}} \text {. }
$$

Daher bedeutet $s_{A B}>1$ nicht notwendigerweise, dass A häufiger hilft als $B$, da es ja so sein kann, dass B seltener Hilfe benötigt $\left(\pi_{B}<\pi_{A}\right)$. Wenn $s_{A B}$ $>1$, dann gilt aber, dass $\mathrm{A}$ relativ zu $\mathrm{B}$ häufiger hilft. Wir unterscheiden also die bedingte Wahr- scheinlichkeit $\alpha_{i}$, dass $i$ hilft, gegeben $j$ benötigt Hilfe, von der marginalen Wahrscheinlichkeit $\alpha_{i} \pi_{j}$, dass $i$ hilft. Mithin gibt $\frac{\alpha_{A} \pi_{B}}{\alpha_{B} \pi_{A}}=s_{A B} \frac{\pi_{B}}{\pi_{A}}$ an, welcher Akteur im IS häufiger hilft. Der Einfachheit halber schreiben wir im Folgenden kurz „ $i$ hilft" anstelle von „ $i$ hilft, gegeben dass $j$ Hilfe benötigt “

Die Erfahrungsgröße spezifiziert, wie oft ein Akteur tatsächlich geholfen hat, wenn der Partner Hilfe benötigte. Wir definieren die Erfahrungsgröße als das Verhältnis der tatsächlich geleisteten Hilfe von A zur tatsächlich geleisteten Hilfe von B. Mit $p_{\mathrm{i}}=$ (Häufigkeit der Hilfeleistungen von i)/(Häufigkeit der Hilfsbedürftigkeit von $j$ ) ist die Erfahrungsgröße also

$$
a_{A B}=\frac{p_{A}}{p_{B}} \text {. }
$$

Ziel- und Erfahrungsgröße müssen nicht übereinstimmen. Es kann ja beispielsweise der Fall sein, dass die beiden Akteure unterschiedliche Zielgrößen haben, so dass die Hilfeleistungen von B der Zielgröße von A nicht entsprechen. Wir modellieren die Diskrepanz zwischen Ziel- und Erfahrungsgröße als:

$$
D=\frac{p_{A}+\alpha_{A}}{p_{B}+\alpha_{B}}-\frac{\alpha_{A}}{\alpha_{B}}
$$

Man beachte, dass $p_{\mathrm{i}}=$ (Häufigkeit der Hilfeleistungen von $i$ )/(Häufigkeit der Hilfsbedürftigkeit von $j$ ) nicht definiert ist, falls $j$ überhaupt noch nicht hilfsbedürftig war. Wir nehmen für diesen Fall $p_{\mathrm{i}}=0$ an. Die Erfahrungsgröße $a_{A B}=\frac{p_{A}}{p_{B}}$ ist nicht definiert, wenn $B$ noch keine Hilfe geleistet hat oder keine Gelegenheit zur Hilfeleistung hatte. Um auch solche Fälle zu berücksichtigen, addieren wir die bedingten Wahrscheinlichkeiten beider Akteure $\left(\alpha_{A}, \alpha_{B}\right)$ in der Erfahrungsgröße. Dabei nehmen wir an, dass die bedingten Wahrscheinlichkeiten dem Kriterium der Pareto-Effizienz genügen (vgl. die Bemerkungen am Ende von Abschnitt 2.2.1). Für $p_{A}, p_{B}>0$ stellt diese Definition der Diskrepanz sicher, dass $D=0$ äquivalent ist mit $\frac{p_{A}}{p_{B}}=\frac{\alpha_{A}}{\alpha_{B}}$.

Wir nehmen an, dass die Akteure danach streben, die Zielgröße $s_{A B}$ mit der Erfahrungsgröße $a_{A B}$ in Einklang zu bringen. Gilt $a_{A B}>s_{A B}$, dann hat Akteur A im früheren Verlauf des IS im Vergleich zu Akteur B häufiger geholfen und wir erwarten, dass B im weiteren Verlauf des IS häufiger hilft, um die Erfahrungs- und die Zielgröße anzugleichen. Dieses Streben von B ist in einem ganz bestimmten Sinn unabhängig vom Verhalten von A: Indem B hilft, kann er zumindest verhindern, dass die Diskrepanz zwischen den beiden Größen weiter zunimmt. Für A ist die Situation umgekehrt: Um die Ziel- und die Erfahrungsgröße in Einklang zu bringen, verweigert 
A seine Hilfe. Dadurch verhindert A, dass die Diskrepanz zwischen den beiden Größen weiter zunimmt.

Matching-Hypothese zur Dynamik wechselseitiger Hilfeleistungen: Je größer die Diskrepanz zwischen der Erfahrungs- und der Zielgröße, desto größer die Wahrscheinlichkeit, dass ein Akteur, der bisher relativ wenig geholfen hat, in der Zukunft helfen wird, und desto kleiner die Wahrscheinlichkeit, dass der Akteur, der bisher relativ viel geholfen hat, in der Zukunft helfen wird.

Man sieht damit auch, in welchem Sinn wir „begrenzt rationales“ Verhalten annehmen: Anreize, nämlich Kosten, Nutzen und Wahrscheinlichkeiten der Hilfsbedürftigkeit, treiben Verhalten, aber unser Modell unterstellt kein spieltheoretisches Gleichgewichtsverhalten; unser Modell verlangt nämlich nicht, dass Verhalten entsprechend unserer Hypothese konsistent ist mit Strategien der Akteure, die ein Nash-Gleichgewicht für das IS sind.

Unsere Hypothese vernachlässigt den (Grenz-)Fall $s_{A B}=a_{A B}$, in dem Ziel- und Erfahrungsgröße übereinstimmen. Außerdem vernachlässigt sie die erste Runde des IS, in der die Akteure noch gar nicht in der Lage sind, Ziel- und Erfahrungsgröße zu vergleichen. Für beide Fälle nehmen wir an, dass derjenige Akteur mit größerer Wahrscheinlichkeit Hilfe leisten wird, der aufgrund der Zielgröße häufiger Hilfe leisten sollte. Diesen Akteur nennen wir auch den schwachen Akteur, während wir seinen Partner auch als den starken Akteur bezeichnen. A ist der schwache und B der starke Akteur, wenn $s_{A B}>1$, also $\alpha_{A}>\alpha_{B}$. A ist der schwache und B der starke Akteur, wenn entweder $\pi_{A}>\pi_{B}$ oder $b_{A}>b_{B}$ oder $c_{A}<c_{B}$, wobei wir voraussetzen, dass sich die beiden Akteure nur in einem der drei Parameter unterscheiden. Der schwache Akteur ist also jener Akteur, der entweder häufiger Hilfe benötigt oder den größeren Verlust fürchtet, falls Hilfe verweigert wird, oder mit geringeren Kosten helfen kann.

Die Zielgröße gibt an, wie sich die bedingten Wahrscheinlichkeiten $\alpha_{A}$ und $\alpha_{B}$ zueinander verhalten, spezifiziert jedoch nicht selbst diese bedingten Wahrscheinlichkeiten. Gelegentlich benötigen wir gleichwohl entsprechende Annahmen über die bedingten Wahrscheinlichkeiten $\alpha_{A}$ und $\alpha_{B}$ (vgl. z. B. oben die Definition der Diskrepanz zwischen Zielund Erfahrungsgröße). Wir werden dann davon ausgehen, dass die bedingten Wahrscheinlichkeiten $\alpha_{A}$ und $\alpha_{B}$ dem Kriterium der Pareto-Effizienz genügen (es gibt keine anderen Werte für die bedingten Wahrscheinlichkeiten, die beide Akteure besser stellen). Daraus folgt dann $\alpha_{i}=1$ für die größere der beiden bedingten Wahrscheinlichkeiten (vgl. Weesie 2006).

Die empirische Prüfung unserer Hypothese setzt voraus, dass wir die Zielgröße, also $\frac{\alpha_{A}}{\alpha_{B}}$, spezifizieren. Diese Spezifikation kann man als das zentrale theoretische Problem unseres Ansatzes sehen. Für symmetrische Akteure liegt ein Equality-Prinzip nahe: Die Akteure helfen sich gleich häufig. Für asymmetrische Akteure ist diese Spezifikation aber keineswegs plausibel. Wir machen hier Gebrauch von klassischen Verteilungstheorien, die auf asymmetrische Akteure angewendet werden können. Zwei paradigmatische Beispiele solcher Theorien sind die in der Sozialpsychologie entwickelte Equity-Theorie und spieltheoretische Verhandlungsmodelle. Wir spezifizieren daher eine Zielgröße, die auf Annahmen der Equity-Theorie beruht, und eine Zielgröße, die durch spieltheoretische Verhandlungsmodelle inspiriert ist und auf der Kalai-Smorodinsky-Lösung beruht. Wir werden beide Zielgrößen miteinander und auch mit dem Equality-Prinzip vergleichen.

\subsubsection{Spezifikation der Zielgröße mit Hilfe der Equity-Theorie}

Verschiedenen Varianten der Equity-Theorie (vgl. Adams 1965; Deutsch 1975, 1985; Walster et al. 1978; Desmarais \& Lerner 1994) liegt die gemeinsame Idee zugrunde, dass Akteure sich durch das Prinzip leiten lassen, dass „Aufwand“ und „Ertrag“ sich proportional zueinander verhalten sollten („distributive Gerechtigkeit“). Varianten dieses Prinzips spielen auch in der Spezifikation von bereits erwähnten modernen und einflussreichen Varianten nicht-egoistischer Nutzenfunktionen (Fehr \& Schmid 1999; Bolton \& Ockenfels 2000; Ockenfels 1999) eine wichtige Rolle. Im Kontext wechselseitiger Hilfeleistungen können wir die Kosten der Hilfeleistung als Aufwand und den Nutzen erhaltener Hilfe als Ertrag auffassen. Für Akteur $i$ repräsentiert $b_{i} \pi_{i} \alpha_{j}$ den erwarteten Nutzen erhaltener Hilfe pro Runde im IS und $c_{i} \pi_{j} \alpha_{i}$ repräsentiert die erwarteten Kosten der Hilfeleistung. Die erwarteten Kosten und Nutzen der Akteure sind proportional zueinander, wenn gilt:

$$
\frac{b_{A} \pi_{A} \alpha_{B}}{c_{A} \pi_{B} \alpha_{A}}=\frac{b_{B} \pi_{B} \alpha_{A}}{c_{B} \pi_{A} \alpha_{B}} .
$$

Wenn wir annehmen, dass die Akteure ihre Zielgröße aufgrund der Equity-Theorie bestimmen, dann ergibt sich mittels einfacher Umformungen die Equity-Zielgröße:

$$
e_{A B}=\frac{\alpha_{A}}{\alpha_{B}}=\frac{\pi_{A}}{\pi_{B}} \times \sqrt{\frac{b_{A} C_{B}}{b_{B} C_{A}}} .
$$

Die Equity-Zielgröße ist eine Funktion von Kosten, Nutzen und Wahrscheinlichkeiten der Hilfsbedürf- 
tigkeit beider Akteure. Die Equity-Zielgröße wächst in $b_{A}, c_{B}$ und $\pi_{A}$ und nimmt in $b_{B}, c_{A}$ und $\pi_{B}$ ab. Akteur A hilft also häufiger als B, wenn der Nutzen und die Wahrscheinlichkeit der Hilfsbedürftigkeit von A höher, die Kosten jedoch geringer sind als die von $\mathrm{B}$.

Es ist offensichtlich, dass die Equity-Theorie für den symmetrischen Fall $\left(c_{A}=c_{B}, \pi_{A}=\pi_{B}, b_{A}=b_{B}\right)$ vorhersagt, dass beide Akteure gleich häufig helfen $\left(e_{A B}=1\right)$. Wenn wir hingegen annehmen, dass die Kosten von $\mathrm{A}\left(c_{A}=8\right)$ geringer sind als die von $\mathrm{B}$ $\left(c_{B}=16\right)$, während die Nutzen $\left(b_{A}=b_{B}=32\right)$ und die Wahrscheinlichkeiten, Hilfe zu benötigen $\left(\pi_{A}=\right.$ $\left.\pi_{B}=0.5\right)$, gleich sind, dann erhalten wir

$$
e_{A B}=\frac{0.5}{0.5} \times \sqrt{\frac{32 \times 16}{32 \times 8}}=\sqrt{2} \text {. }
$$

A sollte also in den Fällen, in denen B Hilfe benötigt, $\sqrt{ } 2$ mal häufiger helfen als $B$ hilft, wenn $A$ Hilfe benötigt.

\subsubsection{Spezifikation der Zielgröße mit Hilfe eines spieltheoretischen Verhandlungsmodells}

Eine theoretische Alternative für die Spezifikation der Zielgröße entwickeln wir aus einem spieltheoretischen Verhandlungsmodell. Es gibt verschiedene solcher Modelle und wir konzentrieren uns auf ein einflussreiches Beispiel, die Kalai-Smorodinsky-Lösung (Kalai \& Smorodinsky 1975; vgl. Roth 1979; Weesie 2006). Verhandlungsmodelle betrachten, grob zusammengefasst, folgendes Problem: Zwei Akteure können eine Übereinkunft aushandeln. In unserem Fall wechselseitiger Hilfeleistungen spezifiziert die Übereinkunft, wie häufig sich die Akteure gegenseitig helfen. Die Übereinkunft definiert also die Zielgröße eines Akteurs. Wenn die Akteure sich nicht einigen, gilt der Status Quo. Im IS wäre der Status Quo das Gleichgewicht, in dem niemals Hilfe geleistet wird. Spieltheoretische Verhandlungsmodelle enthalten mehr oder weniger intuitive Annahmen (Axiome), denen eine Übereinkunft rationaler Akteure genügen muss. Aus diesen Annahmen leitet man die „Lösung“ ab, d. h. die Übereinkunft, die aus diesen Annahmen logisch folgt. ${ }^{10}$ Wir verwenden hier die Kalai-Smorodinsky-Lösung, wollen dabei aber nicht unerwähnt lassen, dass die Vorhersagen, die aus diesem Verhandlungsmodell folgen, sehr hoch mit den Vorhersagen korrelieren, die man bei Verwendung einer anderen klassischen Verhandlungsmodells erhalten würde,

${ }^{10}$ Vgl. Ziegler (1972: Kap. 1) für eine noch immer vorbildliche Diskussion eines solchen axiomatischen Ansatzes und seiner Vorzüge für die soziologische Begriffs- und Theoriebildung. nämlich auf der Grundlage der Nash-Lösung (Nash 1950; vgl. Weesie 2006). ${ }^{11}$ Wir verwenden hier die Kalai-Smorodinsky-Lösung, weil sie zu einer einfacheren Spezifikation der Zielgröße führt.

Angewandt auf unseren Fall besagt die Kalai-Smorodinsky-Lösung, dass die Gewinne aus erhaltener Hilfe relativ zu den maximalen Gewinnen verteilt werden, die die Akteure in allen möglichen Verteilungen erhalten können, die keinen der beiden Akteure schlechter stellen als im Fall des Status Quo. Wenn Akteur A viel gewinnen könnte, während B nur wenig gewinnen könnte, dann folgt aus der Kalai-Smorodinsky-Lösung, dass A viel und $\mathrm{B}$ wenig erhält. Anders als die Herleitung der Equity-Zielgröße, ist die Herleitung der Zielgröße aus der Kalai-Smorodinsky-Lösung komplex (vgl. Weesie 2006). ${ }^{12}$ Für die Parameterkonstellationen, die wir im vorliegenden Beitrag betrachten, impliziert die Kalai-Smorodinsky-Lösung die folgende Verhandlungs-Zielgröße:

$$
k_{A B}=\frac{\alpha_{A}}{\alpha_{B}}=\frac{\pi_{A}}{\pi_{B}} \times \frac{b_{A} c_{B} \pi_{A}+b_{A} b_{B} \pi_{B}}{b_{B} c_{A} \pi_{B}+b_{A} b_{B} \pi_{A}} .
$$

Auch die Verhandlungs-Zielgröße ist eine Funktion der Kosten, der Nutzen und der Wahrscheinlichkeiten, Hilfe zu benötigen. Sie wächst in $b_{A}, c_{B}$ und $\pi_{A}$ und nimmt in $b_{B}, c_{A}$ und $\pi_{B}$ ab. Auch die Verhandlungs-Zielgröße sagt für den symmetrischen Fall $\left(c_{A}=c_{B}, b_{A}=b_{B}, \pi_{A}=\pi_{B}\right)$ voraus, dass beide Akteure gleich häufig helfen $\left(k_{A B}=1\right)$. Betrachten wir nochmals das Beispiel asymmetrischer Akteure mit $c_{A}=8, c_{B}=16, b_{A}=b_{B}=32$ und $\pi_{A}=\pi_{B}=0.5$. In diesem Fall gilt

$$
k_{A B}=\frac{0.5}{0.5} \times \frac{32 \times 16 \times 0.5+32 \times 32 \times 0.5}{32 \times 8 \times 0.5+32 \times 32 \times 0.5}=1.2 \text {. }
$$

A sollte also in den Fällen, in denen B Hilfe benötigt, häufiger helfen als B, wenn A hilfsbedürftig ist. Weitere numerische Beispiele finden sich in der Beschreibung unserer experimentellen Designs.

${ }^{11} \mathrm{Zu}$ beachten ist dabei, dass die Nash-Lösung aus der Verhandlungstheorie etwas anderes ist als ein NashGleichgewicht eines nichtkooperativen Spiels.

12 Die Verwendung der Kalai-Smorodinsky-Lösung für die Spezifikation der Zielgröße widerspricht keineswegs der Tatsache, dass wir ein adaptives Modell begrenzter Rationalität verwenden. Die Kalai-Smorodinsky-Lösung beruht auf Annahmen über rationales Verhalten in nicht wiederholten Verhandlungssituationen, aber aus der Verwendung der Kalai-Smorodinsky-Lösung für die Spezifikation der Zielgröße folgt nicht, dass die Akteure in IS also in einem wiederholten Spiel - Gleichgewichtsstrategien spielen. 


\subsubsection{Equity- und Verhandlungs-Zielgröße im Vergleich}

Wir haben bereits gesehen, dass beide Zielgrößen von den Kosten, Nutzen und Wahrscheinlichkeiten abhängen, Hilfe zu benötigen. Beide Zielgrößen wachsen in $b_{A}, c_{B}$ und $\pi_{A}$ und nehmen in $b_{B}, c_{A}$ und $\pi_{B} \mathrm{ab}$. Beide sagen für den symmetrischen Fall $\left(c_{A}=c_{B}, b_{A}=b_{B}, \pi_{A}=\pi_{B}\right)$ voraus, dass beide Akteure gleich häufig helfen $\left(e_{A B}=k_{A B}=1\right)$. Es mag überraschend erscheinen, dass die Equity- und die Verhandlungs-Zielgrößen ungeachtet unterschiedlicher Annahmen, die ihnen zugrunde liegen, für alle möglichen Parameterkonstellationen auch darin übereinstimmen, welcher der beiden Akteure häufiger helfen sollte, wobei die Equity-Zielgröße für alle möglichen Parameterkonstellationen extremere Werte annimmt als die Verhandlungs-Zielgröße. Es gilt nämlich $e_{A B} \geq k_{A B} \geq 1$ oder aber $e_{A B} \leq k_{A B} \leq 1$ (vgl. Weesie 2006). Für die Bedingungen in unseren Experimenten sind die Unterschiede zwischen beiden Zielgrößen gering. Beide Varianten stimmen tendenziell darin überein, ob ein Akteur relativ „zuviel“ oder „zu wenig“ Hilfe geleistet hat und daher entsprechend der Matching-Hypothese mit geringerer oder größerer Wahrscheinlichkeit Hilfe leisten wird. Die Korrelation zwischen den Diskrepanzen von Equity-Zielgröße und Erfahrungsgröße und von Verhandlungs-Zielgröße und Erfahrungsgröße ist für unsere beiden Experimente 0.973 (Experiment 1) und 0.965 (Experiment 2). Vorhersagen mittels unserer Matching-Hypothese sind daher robust im Hinblick auf die beiden Spezifikationen der Zielgröße. Dies gilt wegen der hohen Korrelation von Vorhersagen mittels der Kalai-Smorodinsky-Lösung mit den Vorhersagen mittels der Nash-Lösung auch im Hinblick auf die Verwendung der Nash-Lösung für die Spezifikation der Zielgröße. ${ }^{13}$

\section{Methoden}

Wir überprüfen unsere Hypothese mit Daten aus zwei Experimenten. Design und Durchführung der Experimente folgen Standardprotokollen der experimentellen Spieltheorie (vgl. Diekmann 2009: 201f.; Camerer 2003: 35ff.). Das Solidaritätsspiel wurde also präsentiert, ohne dass dabei explizit auf „Hilfeleistungen“ und dergleichen Bezug genom-

${ }^{13}$ Es ist eine interessante Frage, ob Spezifikationen der Zielgröße mittels anderer Verteilungstheorien zu wesentlich anderen Vorhersagen führen. Diese Frage sprengt jedoch den Rahmen dieses Beitrags. men wurde. Vielmehr konnten die Versuchpersonen entsprechend den Regeln des Solidaritätsspiels Punkte an andere Versuchspersonen geben bzw. Punkte von anderen Versuchpersonen erhalten. Auf Täuschung der Versuchspersonen wurde verzichtet. Die Experimente waren anreizkompatibel in dem Sinn, dass die Höhe der Entlohnung der Versuchspersonen direkt abhängig war von der Anzahl der erzielten Punkte.

\subsection{Experiment 1}

In Experiment 1 spielten 148 Versuchspersonen (68\% weiblich, Durchschnittsalter 22 Jahre, Standardabweichung 3.6 Jahre) asymmetrische und symmetrische IS. Das Experiment wurde am Computer durchgeführt und mittels z-tree programmiert (Fischbacher 2007). Die Versuchspersonen wurden informiert, dass die Bezahlung am Ende des Experiments vom Verhalten während des Experiments abhing. Für jeden Punkt, den die Versuchspersonen im Experiment verdienten, wurde am Ende des Experiments ein Eurocent ausgezahlt. Zudem erhielten alle Versuchspersonen 5 Euro für die Teilnahme. In Tabelle 1 präsentieren wir Details der verschiedenen experimentellen Bedingungen.

In der linken Spalte werden die verschiedenen experimentellen Bedingungen beschrieben. In den mittleren Spalten spezifizieren wir die Wahrscheinlichkeiten, Hilfe zu benötigen, sowie die Kosten und den Nutzen für beide Akteure. Die beiden rechten Spalten enthalten die entsprechenden Equity- und Verhandlungs-Zielgrößen. Betrachten wir z. B. Bedingung $K_{19}$ : Akteur A benötigt Hilfe mit der Wahrscheinlichkeit $\pi_{A}=0.3$. Die Kosten für geleistete Hilfe betragen für diesen Akteur $c_{A}=8$ Punkte und der Nutzen beträgt $b_{A}=36$ Punkte. Akteur B benötigt Hilfe mit $\pi_{A}=0.7$, also wesentlich häufiger als A. Die Kosten der Hilfeleistung sind für B dreimal höher als für $\mathrm{A}$, nämlich $c_{B}=24$ Punkte. Der Nutzen erhaltener Hilfe entspricht für $\mathrm{B}$ dem von A: $b_{A}=b_{B}=36$ Punkte. Das Experiment enthält eine Bedingung, in der die Akteure symmetrisch sind $\left(K_{11}\right)$, zwei Bedingungen, in denen die Akteure sich in den Kosten unterscheiden $\left(K_{12}\right.$ und $\left.K_{13}\right)$, und zwei Bedingungen, in denen die Akteure sich in den Wahrscheinlichkeiten der Hilfsbedürftigkeit unterscheiden $\left(K_{14}\right.$ und $\left.K_{15}\right)$. Hinzu kommen vier Bedingungen, in denen sich die Akteure in den Wahrscheinlichkeiten und den Kosten unterscheiden: Die Bedingungen $K_{16}$ und $K_{17}$ bezeichnen wir als „Akkumulation ", weil das Solidaritätsspiel in diesen beiden Bedingungen in dem Sinn asymmetrisch ist, dass eine der beiden Versuchspersonen mit zwei 
Tabelle 1 Bedingungen für Experiment 1 und Zielgrößen für jede Bedingung

\begin{tabular}{llllrrrl}
\hline Experimentelle Bedingungen & $\pi_{A}$ & $\pi_{B}$ & $c_{A}$ & $c_{B}$ & $b_{A}=b_{B}$ & $e_{A B}$ & $k_{A B}$ \\
\hline$K_{11}:$ Symmetrie & 0.5 & 0.5 & 8 & 8 & 24 & 1.00 & 1.00 \\
$K_{12}$ : Wenig Asymmetrie in $c$ & 0.5 & 0.5 & 8 & 16 & 32 & 1.41 & 1.20 \\
$K_{13}$ : Viel Asymmetrie in $c$ & 0.5 & 0.5 & 8 & 24 & 36 & 1.73 & 1.36 \\
$K_{14}$ : Wenig Asymmetrie in $\pi$ & 0.6 & 0.4 & 8 & 8 & 24 & 1.50 & 1.23 \\
$K_{15}$ : Viel Asymmetrie in $\pi$ & 0.7 & 0.3 & 8 & 8 & 24 & 2.12 & 1.50 \\
$K_{16}$ : Wenig Akkumulation & 0.6 & 0.4 & 8 & 16 & 32 & 2.60 & 1.74 \\
$K_{17}$ : Viel Akkumulation & 0.6 & 0.4 & 8 & 24 & 36 & 2.33 & 1.56 \\
$K_{18}$ : Wenig Kompensation & 0.3 & 0.7 & 8 & 16 & 32 & 0.61 & 0.77 \\
$K_{19}$ : Viel Kompensation & 0.3 & 0.7 & 8 & 24 & 36 & 0.74 & 0.85 \\
\hline
\end{tabular}

Problemen zugleich konfrontiert wird, nämlich höheren Kosten der eigenen Hilfeleistung und zudem noch einem häufiger hilfsbedürftigen Partner. Die Bezeichnung „Kompensation“ für die Bedingungen $K_{18}$ und $K_{19}$ deutet hingegen an, dass Hilfeleistung für eine der beiden Versuchspersonen mit höheren Kosten verbunden ist, während der Partner seltener Hilfe benötigt. ${ }^{14}$ Man beachte übrigens, dass beide Zielgrößen für jede Bedingung darin übereinstimmen, welcher Akteur häufiger helfen sollte und dass die Equity-Zielgröße für jede Bedingung extremere Werte annimmt - ganz entsprechend unseren Bemerkungen in Abschnitt 2.2.4 über den Vergleich der beiden Zielgrößen.

Jede der neun Sitzungen des Experiments enthielt drei Episoden von 15 Minuten und jede Versuchsperson nahm an jeweils einer Sitzung teil. Während einer Episode spielte jede Versuchsperson in derselben experimentellen Bedingung mit jeweils verschiedenen zufällig zugeordneten Versuchspersonen Solidaritätsspiele. Insgesamt war also jede Versuchsperson drei experimentellen Bedingungen zugeordnet. Eine Episode endete mit dem IS, mit dem die Dauer von 15 Minuten für diese Episode überschritten wurde. Die Fortsetzungswahrscheinlichkeit für ein IS war $w=0.8$ und die Versuchspersonen wussten, dass jedes einzelne IS nach jeder Runde mit einer Wahrscheinlichkeit von 0.2 endete. Vor jedem IS wurden zwei Versuchspersonen einander und den Rollen A oder B zufällig zugeordnet. Die Versuchspersonen konnten ihre Partner nicht identifizieren. Vor jeder Runde eines IS erhielten die

${ }^{14}$ Die Bedingungen $K_{16}-K_{19}$ sind wichtig für die Überprüfung von Vorhersagen, die man aus dem spieltheoretischen Standardansatz ableiten kann (vgl. Abschnitt 2.1 und Vogt \& Weesie 2006). Experiment 1 wurde ursprünglich für die Überprüfung derartiger Vorhersagen entwickelt.
Versuchspersonen eine vorgegebene Anzahl Punkte. Entsprechend unserer Beschreibung des Solidaritätsspiels, konnte Versuchsperson A oder aber Versuchsperson B die gerade erhaltenen Punkte in dieser Runde verlieren. Der Partner hatte dann die Möglichkeit, den Verlust zu verhindern, musste da$\mathrm{zu}$ allerdings eigene Punkte abgeben. Nach jeder Runde erfuhren die Versuchspersonen, ob der Partner bereit war, eigene Punkte abzugeben oder nicht. Diese Informationen blieben während eines IS für beide Versuchspersonen sichtbar.

Am Ende ihrer Sitzung füllten die Versuchspersonen einen kurzen Fragebogen mit einigen demografischen Fragen sowie Fragen zu ihren Einstellungen hinsichtlich verschiedener Aussagen über Vertrauen, Reziprozität, wechselseitige Hilfe usw. aus. Diese Daten werden im vorliegenden Beitrag jedoch nicht verwendet und daher auch nicht weiter besprochen. Eine Sitzung des Experiments dauerte zwischen 70 und 90 Minuten.

\subsection{Experiment 2}

Auch in Experiment 2 spielten die Versuchspersonen Solidaritätsspiele (100 Versuchspersonen, 62\% weiblich, Durchschnittsalter 21 Jahre, Standardabweichung 5.1 Jahre). Wiederum wurde das Experiment am Computer durchgeführt und mit Z-tree programmiert. Die Instruktionen und die Entlohnung der Versuchspersonen entsprachen weitgehend Experiment 1. Der Ablauf des Experiments sowie die Fragebögen am Ende des Experiments unterschieden sich ebenfalls nicht wesentlich von Experiment 1. Ein wesentlicher Unterschied zu Experiment 1 war, dass in Experiment 1 ein unbestimmt oft wiederholtes Solidaritätsspiel verwendet wurde, in Experiment 2 hingegen ein endlich oft wiederholtes Solidaritätsspiel. Die Versuchspersonen wurden 
Tabelle 2 Bedingungen für Experiment 2 und Zielgrößen für jede Bedingung

\begin{tabular}{lcccccc}
\hline Experimentelle Bedingungen & $\pi_{A}$ & $\pi_{B}$ & $c_{A}=c_{b}$ & $b_{A}=b_{B}$ & $e_{A B}$ & $k_{A B}$ \\
\hline$K_{\text {O21 }}$ : Symmetrie & 0.5 & 0.5 & 8 & 24 & 1.00 & 1.00 \\
$K_{T 21}:$ Symmetrie & 0.7 & 0.3 & 8 & 24 & 2.12 & 1.50 \\
$\begin{array}{l}K_{\text {O22: }} \text { : Asymmetrie in } \pi \\
K_{T 22}: \text { Asymmetrie in } \pi\end{array}$ & & & & & & \\
\hline
\end{tabular}

vorab darüber informiert, dass jedes Solidaritätsspiel nach der fünften Runde endete.

In Experiment 2 spielten die Versuchspersonen Solidaritätsspiele, bei denen in jeder Runde einer der beiden Akteure in dem Sinn hilfsbedürftig war, dass ihm ohne Hilfe des Partners ein Verlust drohte („threat“; Bedingungen $K_{T 21}$ und $K_{T 22}$ ). Diese Spiele entsprachen mithin der Hilfesituation in Experiment 1. In Experiment 2 spielten die Versuchspersonen aber auch Solidaritätsspiele, bei denen in jeder Runde einer der beiden Akteure in dem Sinn hilfsbedürftig war, dass er mit Hilfe des Partners eine Anzahl zusätzlicher Punkte gewinnen konnte („opportunity“; Bedingungen $K_{\mathrm{O} 21}$ und $K_{\mathrm{O} 22}$ ). Um diese Punkte zu gewinnen, musste der Partner Punkte abgeben. ${ }^{15}$ In Experiment 2 wurden demnach experimentelle Bedingungen verwendet, die einmal als potenzieller Verlust und einmal als potenzieller Gewinn operationalisiert wurden (Tabelle 2). Jede Versuchsperson nahm an zwölf endlich oft wiederholten Solidaritätsspielen teil, welche gleichmäßig den experimentellen Bedingungen und den Rollen A und B zugeordnet waren. Tabelle 2 kann man entnehmen, dass Asymmetrie ausschließlich in den Wahrscheinlichkeiten, Hilfe zu benötigen, manipuliert wurde.

Für den spieltheoretischen Standardansatz (vgl. Abschnitt 2.1) ist die Annahme wesentlich, dass das Solidaritätsspiel unbestimmt oft wiederholt wird, nicht aber endlich oft, da die spieltheoretische Rationalität in einem endlich oft wiederholten Spiel erzwingt, dass niemals Hilfe geleistet wird (so das bekannte „backward induction“-Argument für endlich oft wiederholte Spiele). Unser adaptives Verhaltensmodell können wir hingegen sowohl auf unbestimmt oft wiederholte Spiele als auch auf endlich oft wiederholte Spiele anwenden. Die hier verfolgte empirische Prüfung des Modells mittels zweier Ex-

15 Experiment 2 wurde ursprünglich entwickelt, um den Einfluss von Risikopräferenzen für Gewinne und Verluste auf prosoziales Verhalten in Solidaritätsspielen mit Gewinn- und Verlustmöglichkeiten zu untersuchen (vgl. Vogt et al. 2006). Im vorliegenden Beitrag abstrahieren wir von solchen Effekten. perimente, die sowohl den Fall der unbestimmt häufigen Wiederholung als auch den des endlich oft wiederholten Spiels abdecken, hat daher auch den Vorteil, dass sie Indizien für die Robustheit des Modells unter unterschiedlichen Anwendungsbedingungen liefert.

\section{Resultate}

Für die Überprüfung unserer Hypothese zur Dynamik wechselseitiger Hilfeleistungen verwenden wir ein logistisches Regressionsmodell mit Zufallseffekten für die Versuchspersonen (random effects model, Maximum Likelihood Schätzung; Snijders \& Bosker 2004: Kap. 11):

Logit(Wahrscheinlichkeit, dass Akteur i in Bedingung $h$ in Runde thilft)

$=\beta_{1} D_{i b t}+\beta_{2} K_{b}+\beta_{3}$ Kontrollvariablen $+u_{i}$.

Mit $D_{i b t}$ notieren wir die Diskrepanz zwischen der Erfahrungsgröße und der Zielgröße von Akteur $i$ in Bedingung $h$ in Runde $t . K_{b}$ gibt die experimentellen Bedingungen an ( $b$ läuft über die verschiedenen experimentellen Bedingungen in Kombination mit der entsprechenden Rolle A oder B, mit Symmetrie als Referenzkategorie). Die Kontrollvariablen repräsentieren die Episoden während einer Sitzung und die wiederholten Spiele während einer Episode. Die Random-Effekt-Terme auf dem Versuchspersonen-Niveau notieren wir mit $u_{i}$.

In Tabelle 3 präsentieren wir die Ergebnisse für Experiment 1 . Wir präsentieren ein Modell für die Equity-Zielgröße (Modell 1) und ein Modell für die Verhandlungs-Zielgröße (Modell 2). In beiden Modellen ist der positive Effekt der Diskrepanz der beiden Zielgrößen auf die Hilfsbereitschaft hoch signifikant und das bestätigt unsere Hypothese. Je größer also die Diskrepanz zwischen der Erfahrungsgröße und der Zielgröße, desto größer ist die Wahrscheinlichkeit, dass der Akteur helfen wird, der bisher relativ wenig geholfen hat.

In zusätzlichen Analysen, die wir hier nicht im Einzelnen präsentieren, haben wir nichtlineare Effekte der Diskrepanz zwischen der Erfahrungs- und Ziel- 
Tabelle 3 Experiment 1, logistisches Regressionsmodell mit Zufallseffekten (random effects model), N(Entscheidungen) $=4683, \mathrm{~N}($ Individuen $)=148,{ }^{* * *}: p<0.01,{ }^{* *}: p<0.05,{ }^{*}: p<0.1$ (zweiseitiger Test)

\begin{tabular}{lcccc}
\hline $\begin{array}{l}\text { Prosoziales } \\
\text { Verhalten }\end{array}$ & $\begin{array}{c}\text { MODELL 1: } \\
\text { Equity } \\
\text { Koeff (se) }\end{array}$ & $\begin{array}{c}\text { MODELL 2: } \\
\text { Verhandlung } \\
\text { Koeff (se) }\end{array}$ & $\begin{array}{c}\text { MODELL 3: } \\
\text { Equity } \\
\text { Koeff (se) }\end{array}$ & $\begin{array}{c}\text { MODELL 4: } \\
\text { Verhandlung } \\
\text { Koeff (se) }\end{array}$ \\
\hline Diskrepanz & $0.415(0.057)^{* * *}$ & $0.695(0.078)^{* * *}$ & $1.369(0.293)^{* * *}$ & $1.374(0.294)^{* * *}$ \\
$\begin{array}{l}\text { Diskrepanz } x \\
\text { schwacher Akteur }\end{array}$ & & $0.404(0.361)$ & $0.227(0.354)$ \\
$\begin{array}{l}\text { Diskrepanz } x \\
\text { starker Akteur }\end{array}$ & & $-1.124(0.299)^{* * *}$ & $-0.942(0.306)^{* * *}$ \\
\hline
\end{tabular}

Referenz: $K_{11}$

(Symmetrie)

Rolle A

\begin{tabular}{|c|c|c|c|c|}
\hline$K_{12 A}: \alpha_{A}>\alpha_{B}$ & $-0.846(0.342)^{* *}$ & $-0.845(0.345)^{* *}$ & $-0.894(0.345)^{* *}$ & $-0.852(0.347)^{* *}$ \\
\hline$K_{13 A}: \alpha_{A}>\alpha_{B}$ & $-1.035(0.254)^{* * *}$ & $-1.030(0.256)^{* * *}$ & $-1.050(0.257)^{* * *}$ & $-1.010(0.257)^{* * *}$ \\
\hline$K_{14 A}: \alpha_{A}>\alpha_{B}$ & $-0.853(0.319) * * *$ & $-0.885(0.321)^{* * *}$ & $-0.991(0.323) * * *$ & $-0.951(0.324)^{* * *}$ \\
\hline$K_{15 A}: \alpha_{A}>\alpha_{B}$ & $0.524(0.191)^{* * *}$ & $0.539(0.192)^{* * *}$ & $0.468(0.195)^{* *}$ & $0.535(0.195)^{* * *}$ \\
\hline$K_{16 A}: \alpha_{A}>\alpha_{B}$ & $-0.885(0.355)^{* *}$ & $-0.881(0.358)^{* *}$ & $-0.901(0.357)^{* *}$ & $-0.872(0.359)^{* *}$ \\
\hline$K_{17 A}: \alpha_{A}>\alpha_{B}$ & $-0.876(0.318)^{* * *}$ & $-0.900(0.321)^{* * *}$ & $-1.106(0.323)^{* * *}$ & $-1.028(0.324)^{* * *}$ \\
\hline$K_{18 A}: \alpha_{B}>\alpha_{A}$ & $-0.855(0.305)^{* * *}$ & $-0.872(0.307)^{* * *}$ & $-0.879(0.307)^{* * *}$ & $-0.898(0.308)^{* * *}$ \\
\hline$K_{19 A}: \alpha_{B}>\alpha_{A}$ & $-0.452(0.257)^{*}$ & $-0.424(0.258)^{*}$ & $-0.480(0.259)^{*}$ & $-0.461(0.259)^{*}$ \\
\hline \multicolumn{5}{|l|}{ Rolle B } \\
\hline$K_{12 B}: \alpha_{A}>\alpha_{B}$ & $-1.376(0.343)^{* * *}$ & $-1.419(0.346)^{* * *}$ & $-1.359(0.345)^{* * *}$ & $-1.391(0.347)^{* * *}$ \\
\hline$K_{13 B}: \alpha_{A}>\alpha_{B}$ & $-1.759(0.261)^{* * *}$ & $-1.817(0.263)^{* * *}$ & $-1.709(0.263)^{* * *}$ & $-1.758(0.265)^{* * *}$ \\
\hline$K_{14 B}: \alpha_{A}>\alpha_{B}$ & $-0.840(0.302)^{* * *}$ & $-0.856(0.304)^{* * *}$ & $-0.830(0.304)^{* * *}$ & $-0.851(0.305)^{* * *}$ \\
\hline$K_{15 B}: \alpha_{A}>\alpha_{B}$ & $-0.765(0.162)^{* * *}$ & $-0.804(0.162)^{* * *}$ & $-0.749(0.162)^{* * *}$ & $-0.780(0.163)^{* * *}$ \\
\hline$K_{16 B}: \alpha_{A}>\alpha_{B}$ & $-2.597(0.367)^{* * *}$ & $-2.637(0.370)^{* * *}$ & $-2.528(0.368)^{* * *}$ & $-2.562(0.371)^{* * *}$ \\
\hline$K_{17 B}: \alpha_{A}>\alpha_{B}$ & $-1.209(0.298) * * *$ & $-1.263(0.301)^{* * *}$ & $-1.210(0.301)^{* * *}$ & $-1.257(0.303)^{* * *}$ \\
\hline$K_{18 B}: \alpha_{B}>\alpha_{A}$ & $-0.812(0.339)^{* *}$ & $-0.860(0.341)^{* *}$ & $-1.108(0.346)^{* * *}$ & $-1.028(0.346)^{* * *}$ \\
\hline$K_{19 B}: \alpha_{B}>\alpha_{A}$ & $-1.097(0.324) * * *$ & $-1.157(0.326)^{* * *}$ & $-1.445(0.340)^{* * *}$ & $-1.384(0.337)^{* * *}$ \\
\hline $\begin{array}{l}\text { Episode } 2 \\
\text { einer Sitzung }\end{array}$ & $0.151(0.116)$ & $0.149(0.117)$ & $0.136(0.118)$ & $0.141(0.118)$ \\
\hline $\begin{array}{l}\text { Episode } 3 \\
\text { einer Sitzung }\end{array}$ & $0.293(0.119)^{* *}$ & $0.294(0.119)^{* *}$ & $0.296(0.120)^{* *}$ & $0.295(0.120)^{* *}$ \\
\hline IS pro Episode & $-0.006(0.015)$ & $-0.007(0.015)$ & $-0.010(0.015)$ & $-0.010(0.015)$ \\
\hline SD (Versuchsperson) & $1.308(0.104)$ & $1.326(0.106)$ & $1.319(0.105)$ & $1.331(0.106)$ \\
\hline Konstante & $0.692(0.216)^{* * *}$ & $0.695(0.217)^{* * *}$ & $0.681(0.217)^{* * *}$ & $0.688(0.218)^{* * *}$ \\
\hline $\mathrm{AIC}$ & 5599.550 & 5571.761 & 5537.704 & 5538.650 \\
\hline Modell Chi ${ }^{2}$ & 211.214 & 233.094 & 252.916 & 253.670 \\
\hline$d f$ & 20 & 20 & 22 & 22 \\
\hline Log-likelihood & -2777.775 & -2763.881 & -2744.852 & -2745.325 \\
\hline
\end{tabular}

größe untersucht, aber die quadratischen Modelle hinsichtlich der Zielgrößen führen nicht zu besseren Gütekriterien. Das gilt auch, wenn wir die Interaktion zwischen den experimentellen Bedingungen für Rolle A oder B und der Diskrepanz berücksichti- gen. Außerdem haben wir untersucht, ob sich der Effekt der Diskrepanz für schwache und starke Akteure unterscheidet. Modell 3 (Equity-Zielgröße) und Modell 4 (Verhandlungs-Zielgröße) in Tabelle 3 präsentieren die Ergebnisse. Wir finden für beide 
Modelle einen hoch signifikanten negativen Effekt der Interaktion zwischen der Diskrepanz und dem starken Akteur, während der Interaktionseffekt zwischen der Diskrepanz und dem schwachen Akteur positiv und nicht signifikant ausfällt. Der Effekt der Diskrepanz ist also für den starken Akteur systematisch schwächer als für den schwachen Akteur. Das kann daran liegen, dass der starke Akteur der Zielgröße zufolge relativ weniger häufig Hilfe leisten sollte und dadurch ein Verhalten des starken Akteurs, das von der Zielgröße abweicht, einen stärkeren Effekt auf die Diskrepanz ausübt. Es kann aber auch daran liegen, dass ein starker Akteur im Allgemeinen durch wechselseitige Hilfeleistungen weniger Gewinn aus dem wiederholten Solidaritätsspiel zieht, wenn es entsprechend den Zielgrößen gespielt wird, da im Vergleich zum schwachen Akteur seine Kosten der Hilfeleistung größer sind, seine Wahrscheinlichkeit der Hilfsbedürftigkeit kleiner oder sein Nutzen aus erhaltener
Hilfe geringer ist. Dadurch hat der starke Akteur im Allgemeinen auch geringere Anreize, Ziel und Erfahrungsgröße in Einklang zu bringen.

In Tabelle 4 präsentieren wir die Ergebnisse für Experiment 2. Die Modelle 5 (Equity-Zielgröße) und 6 (Verhandlungs-Zielgröße) sind mit den entsprechenden Modellen für Experiment 1 vergleichbar. Auch die Daten von Experiment 2 bestätigen unsere Hypothese: Je größer die Diskrepanz zwischen der Erfahrungsgröße und der Zielgröße, desto größer die Wahrscheinlichkeit, dass der Akteur helfen wird, der bisher relativ wenig geholfen hat. Wiederum führen hier nicht im Einzelnen präsentierte quadratische Modelle und Modelle mit Interaktionen zwischen den experimentellen Bedingungen für Rolle A und B und der Diskrepanz zu keinen besseren Gütekriterien. Die Modelle 7 und 8 zeigen auch für Experiment 2, dass der Effekt der Diskrepanz

Tabelle 4 Experiment 2, logistisches Regressionsmodell mit Zufallseffekten (random effects model), N(Entscheidungen) $=3000, \mathrm{~N}$ (Individuen) $=100,{ }^{* *}: p<0.01,{ }^{*}: p<0.05,{ }^{*}: p<0.1$ (zweiseitiger Test)

\begin{tabular}{|c|c|c|c|c|}
\hline Prosoziales Verhalten & $\begin{array}{c}\text { MODELL 5: } \\
\text { Equity } \\
\text { Koeff (se) }\end{array}$ & $\begin{array}{c}\text { MODELL 6: } \\
\text { Verhandlung } \\
\text { Koeff (se) }\end{array}$ & $\begin{array}{c}\text { MODELL 7: } \\
\text { Equity } \\
\text { Koeff (se) }\end{array}$ & $\begin{array}{c}\text { MODELL 8: } \\
\text { Verhandlung } \\
\text { Koeff (se) }\end{array}$ \\
\hline Diskrepanz & $0.597(0.061)^{* * *}$ & $0.899(0.085) * * *$ & $1.195(0.145)^{* * *}$ & $1.195(0.145)^{* * *}$ \\
\hline $\begin{array}{l}\text { Diskrepanz x } \\
\text { schwacher Akteur }\end{array}$ & & & $-0.419(0.322)$ & $-0.288(0.313)$ \\
\hline $\begin{array}{l}\text { Diskrepanz x } \\
\text { starker Akteur }\end{array}$ & & & $-0.741(0.158)^{* * *}$ & $-0.472(0.178) * * *$ \\
\hline \multicolumn{5}{|l|}{$\begin{array}{l}\text { Referenz: } K_{T 21} \\
\text { (Symmetrie, threat) }\end{array}$} \\
\hline$K_{\mathrm{O} 21}:$ Symmetrie (opp.) & $0.076(0.134)$ & $0.080(0.135)$ & $0.082(0.136)$ & $0.082(0.136)$ \\
\hline \multicolumn{5}{|l|}{ Rolle A } \\
\hline$K_{T 22 A}: \alpha_{A}>\alpha_{B}$ & $-0.041(0.185)$ & $-0.008(0.186)$ & $-0.029(0.188)$ & $0.004(0.187)$ \\
\hline$K_{O 22 A}: \alpha_{A}>\alpha_{B}$ & $-0.075(0.180)$ & $-0.062(0.180)$ & $-0.072(0.187)$ & $-0.048(0.184)^{* * *}$ \\
\hline \multicolumn{5}{|l|}{ Rolle B } \\
\hline$K_{T 22 B}: \alpha_{A}>\alpha_{B}$ & $-0.416(0.175)^{* *}$ & $-0.501(0.175)^{* * *}$ & $-0.395(0.175)^{* *}$ & $-0.476(0.175)^{* * *}$ \\
\hline$K_{\mathrm{O} 22 B}: \alpha_{A}>\alpha_{B}$ & $-0.258(0.170)$ & $-0.361(0.169) * *$ & $-0.265(0.169)$ & $-0.354(0.169)^{* *}$ \\
\hline \multicolumn{5}{|l|}{ Episode 2} \\
\hline einer Sitzung & $0.001(0.083)$ & $0.001(0.084)$ & $0.007(0.084)$ & $0.005(0.084)$ \\
\hline IS pro Episode & $-0.082(0.024) * * *$ & $-0.079(0.025)^{* * *}$ & $-0.075(0.025) * * *$ & $-0.074(0.025)^{* * *}$ \\
\hline SD(Teilnehmer) & $1.148(0.110)$ & $1.159(0.111)$ & $1.163(0.111)$ & $1.162(0.111)$ \\
\hline Konstante & $0.587(0.173)^{* * *}$ & $0.560(0.174) * * *$ & $0.532(0.175)^{* * *}$ & $0.531(0.175)^{* * *}$ \\
\hline$\overline{A I C}$ & 3680.606 & 3663.442 & 3661.151 & 3660.361 \\
\hline Modell Chi ${ }^{2}$ & 125.181 & 139.775 & 144.342 & 144.679 \\
\hline$d f$ & 8 & 8 & 10 & 10 \\
\hline Log-likelihood & -1830.303 & -1821.721 & -1818.575 & -1818.181 \\
\hline
\end{tabular}


für den starken Akteur systematisch schwächer ausfällt als für den schwachen Akteur. ${ }^{16}$

In einer explorierenden Analyse gehen wir abschließend der Frage nach, ob Modelle mit der EquityZielgröße oder Modelle mit der Verhandlungs-Zielgröße die Daten besser organisieren. Es geht uns nun also um einen quantitativen Vergleich. Wir verwenden das Informationskriterium AIC von Akaike (1973; vgl. Burnham \& Anderson 2002). Die AICWerte der beiden Modelle sind ähnlich, was wegen der hohen Korrelation der Equity- und der Verhandlungs-Diskrepanz Variablen $(0.973$ in Experiment 1 und 0.965 in Experiment 2) nicht verwundert. Dennoch gibt es Indizien, dass die Verhandlungs-Zielgröße die Daten etwas besser organisiert. Betrachten wir zunächst die Modelle ohne Interaktionseffekte für Experiment 1. Das Modell für die Verhandlungs-Zielgröße (M2, 5572) beschreibt die Daten besser als das Modell für die Equity-Zielgröße (M1, 5600). Der Effekt der Diskrepanz auf den starken Akteur ist für das Modell mit der Verhandlungs-Zielgröße etwas schwächer als für das Modell mit der Equity-Zielgröße, während zugleich das Modell mit der VerhandlungsZielgröße die Daten wiederum etwas besser beschreibt. Zusätzlich betrachten wir als BaselineModell ein Modell für das Equality-Prinzip, also die Annahme, dass beide Akteure gleich häufig Hilfe leisten. Auch bei Asymmetrie gilt unter dem Equality-Prinzip $s_{A B}=1$. Es ist auffällig, dass nicht nur das AIC des Modells 2 mit der VerhandlungsZielgröße (5572) geringer ist als das AIC des Modells 1 mit der Equity-Zielgröße (5600), sondern dass auch das AIC des Modells für das EqualityPrinzip (5591) einen geringeren Wert annimmt als das AIC des Modells mit der Equity-Zielgröße. Die Tatsache, dass auch das Modell für das EqualityPrinzip zu besseren Vorhersagen führt als das Modell mit der Equity-Zielgröße, unterstützt unsere Vermutung, dass das Modell mit der Equity-Zielgröße für asymmetrische Spiele zu extreme Zielgrößen impliziert. Wir finden ähnliche Ergebnisse für

\footnotetext{
${ }^{16}$ In Experiment 2 finden wir die bekannten (z. B. Andreoni \& Miller 1993) Endspieleffekte in endlich oft wiederholten sozialen Dilemmata: Das wiederholte Solidaritätsspiel beginnt mit relativ viel Hilfeleistungen, die zum Ende des wiederholten Spiels hin immer seltener werden (vgl. Vogt et al. 2006). Unser adaptives Modell kann solche Endspieleffekte ersichtlich nicht erklären, jedenfalls nicht ohne zusätzliche Annahmen. Der spieltheoretische Standardansatz hat das in gewisser Weise komplementäre Problem, dass man ohne zusätzliche Annahmen nicht erklären kann, dass in einem endlich oft wiederholten Solidaritätsspiel überhaupt Hilfe geleistet wird.
}

Experiment 2: Ein Vergleich der Modelle ohne Interaktionseffekte (M5, 3681; M6, 3663) wie auch der Modelle mit Interaktionseffekten (M7, 3661; M8, 3660) zeigt, dass die Modelle mit der Verhandlungs-Zielgröße die Daten besser organisieren. Allerdings ist das AIC des Modells für das EqualityPrinzip in Experiment 2 (3656) auch kleiner als das AIC des Modells mit der Verhandlungs-Zielgröße.

\section{Diskussion}

Im vorliegenden Beitrag haben wir an einem Beispiel prosozialen Verhaltens gezeigt, wie man die experimentelle Spieltheorie in der Soziologie anwenden kann. Wir haben die Dynamik wechselseitiger Hilfeleistungen zwischen asymmetrischen Akteuren untersucht. Damit verbinden wir die Untersuchung von Bedingungen der Kooperation unter Anreizproblemen mit der Untersuchung von Effekten sozialer Ungleichheit auf individuelles Verhalten. Standardmodelle der Spieltheorie stoßen bei der Analyse der Dynamik wechselseitiger Hilfeleistungen zwischen asymmetrischen Akteuren an ihre Grenzen. Wir haben Ideen aus der experimentellen Spieltheorie für die Entwicklung eines alternativen Modells verwendet. Es handelt sich um ein adaptives Modell. Wir nehmen an, dass das Verhalten der Akteure von zwei Größen abhängt. Die eine dieser beiden Größen nennen wir die Zielgröße. Sie gibt an, wie oft ein Akteur Hilfe leisten möchte, falls der Partner Hilfe benötigt. Demgegenüber spezifiziert die Erfahrungsgröße, wie oft ein Akteur in Situationen, in denen der Partner Hilfe benötigte, de facto Hilfe geleistet hat. Wir nehmen adaptives Verhalten an, das auf einem Matching-Mechanismus basiert: Die Akteure streben danach, die Zielgröße mit der Erfahrungsgröße in Einklang zu bringen. Das zentrale Problem eines solchen Ansatzes ist die Spezifikation der Zielgröße. Wir haben zwei theoretische Varianten für die Ableitung der Zielgröße unterschieden: Equity-Theorie und ein spieltheoretisches Verhandlungsmodell (Kalai-Smorodinsky-Lösung). Das adaptive Modell ist robust in dem Sinne, dass beide Varianten für die Ableitung der Zielgröße zu ähnlichen Vorhersagen für die Dynamik wechselseitiger Hilfeleistungen führen. Unsere Hypothese haben wir mittels experimenteller Daten überprüft. Es zeigt sich, dass die Diskrepanz zwischen der Zielgröße und der Erfahrungsgröße in der Tat das Verhalten der Akteure beeinflusst. Darüber hinaus gibt es einige Anzeichen, wenn auch keine sehr starke Evidenz, dass die verhandlungstheoretische Variante der stärker sozialpsycholo- 
gisch orientierten Variante der Equity-Theorie hinsichtlich der unterschiedlichen quantitativen Vorhersagen überlegen ist.

Aus dieser Untersuchung ergeben sich eine Reihe vielversprechender Anknüpfungspunkte und Fragen für die weitere Forschung. Im Allgemeinen wäre weitere experimentelle Forschung nützlich. Wir haben gesehen, dass robuste qualitative Vorhersagen unseres adaptiven Verhaltensmodells empirisch bestätigt werden. Unter den Bedingungen unserer beiden Experimente ähneln sich die Equity- und die Verhandlungs-Zielgröße. Dadurch wird es schwierig, mit empirischen Daten zwischen den quantitativen Vorhersagen der beiden Modellvarianten zu unterscheiden. Es wäre ratsam, ein experimentelles Design mit Bedingungen für die Kosten und den Nutzen der Hilfeleistung, sowie Wahrscheinlichkeiten der Hilfsbedürftigkeit zu entwickeln, unter denen sich die Equity- und die Verhandlungs-Zielgröße stärker unterscheiden. Das würde es erleichtern, die Frage zu beantworten, welche der beiden Spezifikationen der Zielgröße empirisch adäquater ist.

Im Hinblick auf die weitere Theoriebildung möchten wir drei Richtungen skizzieren. Die erste betrifft die Schnittstelle der Erforschung von Kooperationsproblemen und sozialer Ungleichheit. Unser Beitrag behandelt Effekte sozialer Ungleichheit für individuelles Verhalten bei Kooperationsproblemen - Ungleichheit ist in unserem Modell exogen gegeben. Individuelles Verhalten bei solchen Problemen - in unserem Fall also Hilfeleistungen oder deren Verweigerung - kann aber wiederum Effekte für die Dynamik sozialer Ungleichheit haben. Unter welchen Bedingungen nimmt durch Hilfeleistungen oder deren Verweigerung die Ungleichheit zwischen den Akteuren zu oder ab? Für die Untersuchung solcher Fragen und für die Endogenisierung sozialer Ungleichheit kann man das iterierte Solidaritätsspiel in der Weise erweitern, dass Hilfeleistungen bzw. verweigerte Hilfeleistungen in Runde $t$ die Anreizstruktur des Spiels in der folgenden Runde $t+1$ beeinflussen, also Effekte für die Wahrscheinlichkeiten haben, mit denen die Akteure in der Zukunft hilfsbedürftig werden, und für ihre Kosten der Hilfeleistung bzw. den Nutzen erhaltener Hilfe. Die Analyse eines solchen dynamischen Modells ist keineswegs trivial.

Eine zweite Frage für die zukünftige Theoriebildung betrifft weitere Alternativen zum spieltheoretischen Standardansatz. Unser Modell gibt die Annahme spieltheoretischen Gleichgewichtsverhaltens auf und ersetzt sie durch eine adaptive Verhaltensannahme. Wir haben darauf hingewiesen, dass andere Modelle der experimentellen Spieltheorie an der Gleichgewichtsannahme festhalten und andere Standardannahmen der Spieltheorie ersetzen. Es wäre daher naheliegend, das iterierte Solidaritätsspiel auch in einem solchen Modellrahmen zu analysieren, also beispielsweise zu untersuchen, welche Vorhersagen aus einem Modell der Ungleichheitsaversion (Fehr \& Schmidt 1999; Bolton \& Ockenfels 2000) gewonnen werden können. Das würde die Lösung von zwei Problemen erfordern: Zunächst ist es so, dass die Modelle zur Ungleichheitsaversion typisch annehmen, dass die Akteure symmetrisch (im Sinne von Abschnitt 2) sind. Wie unser Beitrag gerade zeigt, ist es aber keineswegs trivial, Ungleichheitsaversion für asymmetrische Akteure überhaupt zu konzeptualisieren. Das zweite Problem besteht dann darin, ein Modell der Ungleichheitsaversion nicht nur auf ein einmalig gespieltes Solidaritätsspiel anzuwenden, sondern gerade auch auf das iterierte Solidaritätsspiel. Typische Anwendungen von Modellen der Ungleichheitsaversion betreffen nichtiterierte Spiele. Die Anwendung auf iterierte Spiele ist nicht nur in theoretischer Hinsicht herausfordernd. In empirischer Hinsicht ergibt sich bei der Anwendung auf iterierte Spiele ersichtlich die Schwierigkeit, die Effekte „schwacher“ Reziprozität, also bedingter Kooperation, von denen „starker“ Reziprozität auf der Grundlage nicht ausschließlich eigennütziger Motiven zu separieren.

Eine dritte Möglichkeit weiterer Theoriebildung betrifft die Integration des spieltheoretischen Standardansatzes und unseres adaptiven Verhaltensmodells. Der spieltheoretische Standardansatz zur Analyse prosozialen Verhaltens in einem asymmetrischen sozialen Dilemma leidet unter dem Problem der Gleichgewichtsauswahl: Es gibt viele verschiedene Gleichgewichte, einschließlich vieler Gleichgewichte, die dem Kriterium der Pareto-Effizienz genügen. Der Nachteil unseres Ansatzes aus der experimentellen Spieltheorie ist demgegenüber, dass Pareto-Effizienz im Prinzip nicht berücksichtigt wird. Unser Ansatz richtet sich auf das Verteilungsproblem im Hinblick auf gegenseitige Hilfeleistungen, und die Zielgröße spezifiziert lediglich das Verhältnis der bedingten Wahrscheinlichkeiten, mit denen die Akteure einander helfen, falls der Partner Hilfe benötigt. Sind jedoch beide bedingten Wahrscheinlichkeiten kleiner als 1 , dann wäre es eine $\mathrm{Pa}$ reto-Verbesserung, wenn beide Akteure einander häufiger helfen würden. Das wiederum könnte geschehen, ohne die Zielgröße zu verändern. Unter diesem Gesichtspunkt wäre ein theoretisches Modell interessant, in dem die Akteure neben dem Einklang der Zielgröße und der Erfahrungsgröße auch Pareto-Effizienz anstreben. Man könnte dabei an 
ein Modell denken, in dem zunächst die Pareto-effizienten Gleichgewichte in Trigger-Strategien als „Lösungskandidaten“ in Betracht kommen, um danach einen verhandlungstheoretischer Ansatz wie den von Kalai-Smorodinsky oder auch die EquityTheorie zu verwenden, um das Gleichgewicht zu bestimmen, das mit der entsprechenden Zielgröße konsistent ist, und so das Problem der Gleichgewichtsauswahl zu lösen. ${ }^{17}$

\section{Literatur}

Adams, J.S., 1965: Inequity in Social Exchange. S. 267299 in: L. Berkowitz (Hrsg.), Advances in Experimental Social Psychology, Vol. 2. New York: Academic Press.

Akaike, H., 1973: Information Theory and an Extension of the Maximum Likelihood Principle. S. 267-281 in: B.N. Petrov \& F. Csaki (Hrsg.), Second International Symposium on Information Theory. Budapest: Akademiai Kiado.

Andreoni, J. \& J.H. Miller, 1993: Rational Cooperation in the Finitely Repeated Prisoner's Dilemma: Experimental Evidence. Economic Journal 100: 464-477.

Axelrod, R., 1984: The Evolution of Cooperation. New York: Basic Books.

Bierhoff, H.W., 2002: Prosoziales Verhalten. S. 319-352 in: W. Stroebe, K. Jonas \& M. Hewstone (Hrsg.), Sozialpsychologie, 4. Aufl. Berlin: Springer.

Blau, P.M., 1963: The Dynamics of Bureaucracy, 2. Aufl. Chicago: University of Chicago Press.

Blau, P.M., 1964: Exchange and Power in Social Life. New York: Wiley.

Blau, P.M., 1968: Social Exchange. S. 452-457 in: D.L. Sills (Hrsg.), International Encyclopedia of the Social Sciences, Vol.7. New York: Free Press.

Bolton, G.E. \& A. Ockenfels, 2000: ERC: A Theory of Equity, Reciprocity and Competition. American Economic Review 90: 166-193.

Burnham, K.P. \& D.R. Anderson, 2002: Model Selection and Multimodel Inference: A Practical InformationTheoretic Approach. New York: Springer.

Camerer, C., 2003: Behavioral Game Theory: Experiments in Strategic Interaction. Princeton: Princeton University Press.

Desmarais, S. \& M.J. Lerner, 1994: Entitlements in Close Relationships: A Justice-Motive Analysis. S. 43-63 in: M.J. Lerner \& G. Mikula (Hrsg.), Entitlement and the Affectional Bond: Justice in Close Relationships. New York: Plenum.

Deutsch, M., 1975: Equity, Equality, and Need: What Determines Which Value Will Be Used as the Basis of Distributive Justice? Journal of Social Issues 31: 137-149.

Deutsch, M., 1985: Distributive Justice. New Haven: Yale University Press.

${ }^{17}$ Erste Schritte in diese Richtung unternimmt Weesie 2006.
Diekmann, A., 2004: The Power of Reciprocity. Journal of Conflict Resolution 48: 487-505.

Diekmann, A., 2009: Spieltheorie. Einführung, Beispiele, Experimente. Reinbek: Rowohlt.

Emerson, R.M., 1976: Social Exchange Theory. Annual Review of Sociology 2: 335-362.

Falk, A. \& J.J. Heckman, 2009: Lab Experiments Are a Major Source of Knowledge in the Social Sciences. Science 326: 535-538.

Fehr, E. \& H. Gintis, 2007: Human Motivation and Social Cooperation: Experimental and Analytical Foundations. Annual Review of Sociology 33: 43-64.

Fehr, E. \& K. Schmidt, 1999: A Theory of Fairness, Competition, and Cooperation. Quarterly Journal of Economics 114: 817-868.

Fischbacher, U., 2007: z-tree - Zurich Toolbox for Readymade Economic Experiments. Experimental Economics 10: 171-178.

Fudenberg, D. \& E.S. Maskin, 1986: The Folk Theorem in Repeated Games with Discounting or with Incomplete Information. Econometrica 54: 533-554.

Gächter, S. \& C. Thöni, 2011: Micromotives, Microstructure, and Macrobehavior: The Case of Voluntary Cooperation. Journal of Mathematical Sociology 35: 2665 .

Gouldner, A.W., 1960: The Norm of Reciprocity: A Preliminary Statement. American Sociological Review 25: 161-178.

Hegselmann, R., 1994: Solidarität in einer egoistischen Welt - eine Simulation. S. 349-390 in: J. Nida-Rümelin (Hrsg.), Praktische Rationalität - Grundlagen und ethische Anwendungen des Rational-Choice Paradigmas. Berlin: De Gruyter.

Herrnstein, R.J., 1997: The Matching Law, Papers in Psychology and Economics. New York: Russell Sage.

Kalai, E. \& M. Smorodinsky, 1975: Other Solutions to Nash's Bargaining Problem. Econometrica 45: 16231630.

Kollock, P., 1998: Social Dilemmas: The Anatomy of Cooperation. Annual Review of Sociology 24: 183-214.

Macy, M.W. \& A. Flache, 2002: Learning Dynamics in Social Dilemmas. PNAS 99: 7229-7236.

Nash, J., 1950: The Bargaining Problem. Econometrica 18: 155-162.

Ockenfels, A., 1999: Fairness, Reziprozität und Eigennutz. Tübingen: Mohr.

Rapoport, A., 1974: Introduction. S. 1-14 in: A. Rapoport (Hrsg.), Game Theory as a Theory of Conflict Resolution. Dordrecht: Reidel.

Raub, W. \& V. Buskens, 2006: Spieltheoretische Modelle und empirische Anwendungen in der Soziologie. S. 560-598 in A. Diekmann (Hrsg.), Methoden der Sozialforschung. Kölner Zeitschrift für Soziologie und Sozialpsychologie, Sonderheft 44. Wiesbaden: VS.

Raub, W. \& V. Buskens, 2008: Theory and Empirical Research in Analytical Sociology: The Case of Cooperation in Problematic Social Situations. Analyse \& Kritik 30: 680-722.

Raub, W. \& T. Voss, 1986: Die Sozialstruktur der Kooperation rationaler Egoisten. Zur „utilitaristischen“ Er- 
klärung sozialer Ordnung. Zeitschrift für Soziologie 15: 309-323.

Roth, A.E., 1979: Axiomatic Models of Bargaining. Berlin: Springer.

Snijders, T.A.B. \& R. Bosker, 2004: Multilevel Analysis: An Introduction to Basic and Advanced Multilevel Modeling. London: Sage.

Taylor, M., 1987: The Possibility of Cooperation. Cambridge: Cambridge University Press.

Vogt, S. \& J. Weesie, 2004: Social Support among Heterogeneous Partners. Analyse \& Kritik 26: 398-422.

Vogt, S. \& J. Weesie, 2006: Social Support among Heterogeneous Partners. An Experimental Test. Journal of Economic Interaction and Coordination 1: 215-232.

Vogt, S., J. Weesie \& W. Raub, 2006: Social Support in Opportunity and Threat Situations. Iscore Paper No. 237, Utrecht University.
Voss, T., 1985: Rationale Akteure und soziale Institutionen. München: Oldenbourg.

Walster, E., W.G. Walster \& E. Berscheid, 1978: Equity. Theory and Research. Boston, MA: Allyn and Bacon.

Weesie, J., 1988: Mathematical Models for Competition, Cooperation, and Social Networks. Utrecht: Diss. Utrecht University.

Weesie, J., 2006: Bargaining in Support Games. Iscore Paper No. 242, Utrecht University.

Weesie, J., S. Vogt, V. Buskens \& W. Raub, 2007: Support in an Asymmetric Social Dilemma. S. 155-180 in E. Poppe \& M. Verkuyten (Hrsg.), Culture and Conflict. Liber Amicorum for Louk Hagendoorn. Amsterdam: Aksant.

Ziegler, R., 1972: Theorie und Modell. Der Beitrag der Formalisierung zur soziologischen Theoriebildung. München: Oldenbourg.

\section{Autorenvorstellung}

Sonja Vogt, geb. 1976 in Marsberg. Studium der Soziologie, Philosophie und Linguistik in Düsseldorf. Promotion in Utrecht. Von 2008 bis 2010 Visiting Fellow und seit 2010 Wissenschaftliche Mitarbeiterin am Institut für Volkswirtschaftslehre und am Laboratory for Social and Neural Systems Research der Universität Zürich.

Forschungsschwerpunkte: Soziale Präferenzen, Asymmetrie in sozialen Dilemmata, Endogene Präferenzen.

Wichtigste Publikationen: Social Support among Heterogeneous Partners (mit J. Weesie), Analyse \& Kritik 26, 2004; Social Support among Heterogeneous Partners. An Experimental Test (mit J. Weesie), Journal of Economic Interaction and Coordination 1, 2006; Support in an Asymmetric Social Dilemma (mit J. Weesie, V. Buskens \& W. Raub), in E. Poppe \& M. Verkuyten (Hrsg.), Culture and Conflict. Liber Amicorum for Louk Hagendoorn. Amsterdam.

Werner Raub, geb. 1953 in Essen. Studium der Sozialwissenschaften in Bochum. Promotion in Utrecht. Seit 1996 Professor für Theoretische Soziologie in Utrecht und am Interuniversity Center for Social Science Theory and Methodology (ICS).

Forschungsschwerpunkte: Soziologische Theorie, Soziale Netzwerke, Anwendungen mathematischer Modelle in der Soziologie, Experimentelle Spieltheorie.

Neuere Publikationen: Trust in triads: an experimental study (mit V. Buskens \& J. van der Veer), Social Networks 32, 2010; Micro-Macro Links and Microfoundations (Hrsg. mit M. van Assen \& V. Buskens), Triple Special Issue of Journal of Mathematical Sociology 35, 2011; Micro-Macro Links and Microfoundations in Sociology (mit V. Buskens \& M. van Assen), Journal of Mathematical Sociology 35, 2011; Rational Choice Research on Social Dilemmas (mit V. Buskens), erscheint in: R. Wittek, T.A.B. Snijders \& V. Nee (Hrsg.), Handbook of Rational Choice Social Research.

Jeroen Weesie, geb. 1958 in Amsterdam. Studium der Mathematik und Soziologie in Utrecht. Promotion in Utrecht. Seit 1995 Universitätshauptdozent (Associate Professor) in Utrecht und am Interuniversity Center for Social Science Theory and Methodology (ICS).

Forschungsschwerpunkte: Mathematische Soziologie, Statistische Modellierung, Computersimulation.

Neuere Publikationen: The Rationale of Rationality (mit V. Buskens \& C. Snijders), Rationality and Society 21, 2009; Inequality and procedural justice in social dilemmas (mit O. Aksoy), Journal of Mathematical Sociology 33, 2009; Online Programming Markets (mit C. Snijders), in: K.S. Cook, C. Snijders, V. Buskens \& C. Cheshire (Hrsg.), eTrust. Forming Relationships in the Online World, New York 2009; Beliefs about the Social Orientations of Others (mit O. Aksoy), Journal of Experimental Social Psychology (im Erscheinen).

Vincent Buskens, geb. 1968 in Schimmert (NL). Studium der Mathematik und Sozialwissenschaften in Eindhoven. Promotion in Utrecht. Seit 2004 als Universitätshauptdozent (Associate Professor) und seit 2011 als Professor für Theoretische Soziologie in Utrecht und am Interuniversity Center for Social Science Theory and Methodology (ICS).

Forschungsschwerpunkte: Soziale Netzwerke, Mathematische Soziologie und Computersimulation, Spieltheoretische Modelle in den Sozialwissenschaften.

Neuere Veröffentlichungen: Dynamics of Networks If Everyone Strives for Structural Holes (mit A. van de Rijt), American Journal of Sociology 114, 2008; eTrust: Forming Relationships in the Online World (Hrsg. mit K.S. Cook, C. Snijders \& C. Cheshire), New York: Russell Sage; Micro-Macro links and Microfoundations in Sociology (mit W. Raub \& M. van Assen), Journal of Mathematical Sociology 35, 2011; Rational Choice Research on Social Dilemmas (mit W. Raub), erscheint in: R. Wittek, T.A.B. Snijders \& V. Nee (Hrsg.), Handbook of Rational Choice Social Research. 